\title{
The role of fluctuation-dissipation dynamics in setting initial conditions for inflation
}

\author{
Mar Bastero-Gil ${ }^{a}$ Arjun Berera ${ }^{b}$ Robert Brandenberger ${ }^{c}$ Ian G. \\ Moss $^{d}$ Rudnei O. Ramos ${ }^{e, c}$ João G. Rosa ${ }^{(f)}$ \\ ${ }^{a}$ Departamento de Física Teórica y del Cosmos, Universidad de Granada, Granada-18071, \\ Spain \\ ${ }^{b}$ School of Physics and Astronomy, University of Edinburgh, Edinburgh, EH9 3FD, United \\ Kingdom \\ ${ }^{c}$ Physics Department, McGill University, Montreal, QC, H3A 2T8, Canada \\ ${ }^{d}$ School of Mathematics, Statistics and Physics, Newcastle University, Newcastle upon Tyne, \\ NE1 7RU, United Kingdom \\ ${ }^{e}$ Departamento de Física Teórica, Universidade do Estado do Rio de Janeiro, 20550-013 Rio \\ de Janeiro, RJ, Brazil \\ ${ }^{f}$ Departamento de Física da Universidade de Aveiro and CIDMA, Campus de Santiago, \\ 3810-183 Aveiro, Portugal \\ E-mail: mbg@ugr.es, ab@ph.ed.ac.uk,rhb@physics.mcgill.ca, ian.moss@ncl.ac.uk, \\ rudnei@uerj.br, joao.rosa@ua.pt
}

\begin{abstract}
.
We study the problem of initial conditions for slow-roll inflation along a plateau-like scalar potential within the framework of fluctuation-dissipation dynamics. We consider, in particular, that inflation was preceded by a radiation-dominated epoch where the inflaton is coupled to light degrees of freedom and may reach a near-equilibrium state. We show that the homogeneous field component can be sufficiently localized at the origin to trigger a period of slow-roll if the interactions between the inflaton and the thermal degrees of freedom are sufficiently strong and argue that this does not necessarily spoil the flatness of the potential at the quantum level. We further conclude that the inflaton can still be held at the origin after its potential begins to dominate the energy balance, leading to a period of thermal inflation. This then suppresses the effects of nonlinear interactions between the homogeneous and inhomogeneous field modes that could prevent the former from entering a slow-roll regime. Finally, we discuss the possibility of an early period of chaotic inflation, at large field values, followed by a first stage of reheating and subsequently by a second inflationary epoch along the plateau about the origin. This scenario could prevent an early overclosure of the Universe, at the same time yielding a low tensor-to-scalar ratio in agreement with observations.
\end{abstract}

Keywords: inflation, initial conditions, fluctuation-dissipation dynamics

ArXiv ePrint: 1612.04726 


\section{Contents}

1 Introduction $\quad 1$

2 Dynamical evolution of the inflaton field coupled to a radiation bath 4

3 General Consistency Conditions Analysis $\quad 8$

3.1 Radiative Corrections and the Flatness of the Inflaton Potential 9

$\begin{array}{lll}3.2 & \text { Kinetic and gradient energies before inflation } & 10\end{array}$

$\begin{array}{lll}3.3 & \text { Thermalization and equipartition of energy } & 13\end{array}$

4 Initial Condition Problem for Plateau-Like Inflaton Potentials: An explicit example 14

4.1 Evolution after inflaton-radiation equality, thermal inflation and nonlinear effects 16

4.2 An initial period of chaotic inflation for large field amplitudes followed by $\begin{array}{ll}\text { inflation on the plateau } & 20\end{array}$

5 Conclusions $\quad 22$

A Inflation with the Higgs-like symmetry breaking potential 25

B Non-renormalizable plateau-like potential $\quad 28$

$\begin{array}{lll}\text { B.1 Plateau-like potential with sextic power } & 28\end{array}$

B.2 General non-renormalizable plateau-like potentials 31

B.3 Non-renormalizable plateau model in warm inflation 33

\section{Introduction}

The inflationary scenario [1] is the current paradigm of early Universe cosmology. It addresses a number of problems of Standard Big Bang cosmology, provided the first mechanism based on causal physics to generate the observed density fluctuations on cosmological scales [2], and made predictions concerning the spectrum of cosmic microwave background (CMB) anisotropies which were subsequently successfully verified by the measurements from both WMAP [3] and Planck [4] satellites (among these measurements relevant to inflation, one can mention the near Gaussianity of the fluctuations, the acoustic oscillations in the angular power spectrum of CMB anisotropies, and the small red tilt of the spectrum of fluctuations $)^{1}$.

The inflationary scenario assumes that the patch of the universe we observe underwent a period of almost exponential expansion during some time interval at some very early time. This accelerated expansion is driven by the potential energy of a slowly rolling scalar matter field. A key question when discussing the success of inflation is the initial condition question, namely how likely are initial conditions which lead to a sufficiently large region to enter a period of inflation. There have been claims in the literature that the initial conditions

\footnotetext{
${ }^{1}$ Note, however, that there are alternative early universe scenarios such as the Ekpyrotic scenario [5], String Gas Cosmology [6] and the Matter Bounce model [7, 8], which also lead to fluctuations that are consistent with the current data.
} 
to obtain inflation have to be severely fine tuned [9-12]. Indeed, for small field models of inflation, the initial field velocity has to be severely fine tuned in order to obtain inflation [13].

On the other hand, for large field models of inflation the slow roll trajectory of the scalar field is a local attractor in initial conditions space. This was indicated by the initial studies done in Ref. [14], worked out in more detail in Ref. [15], and generalized to the presence of linear gravitational fluctuations in Ref. [16] (see also Ref. [17] for a recent review). These works used somewhat intuitive reasonings, but investigations using numerical general relativity [18] confirmed the basic picture, and recent numerical work shown in the Refs. [19, 20] contributed to an improved characterization of the attractor basin of large field inflation.

Such large field models tend, however, to predict a too large fraction of tensor modes in the primordial perturbation spectrum, and are thus in tension with the most recent CMB observations by the WMAP and Planck satellites. Observational data seems to suggest, in fact, scalar potentials with a plateau-like region for which the tensor-to-scalar ratio is suppressed. This poses a significant challenge for inflationary model-building, since a slowroll solution is not a phase space attractor for most potentials in this class. This means that inflation can only be triggered if the field is initially located in the inflationary plateau and if its velocity is sufficiently small. In addition, this must be true on super-Hubble scales, since nonlinear effects (both in the field and in the metric) could prevent the start of inflation. This means, in particular, that even when the conditions leading to inflation are satisfied within a Hubble-sized patch, if the field value outside this region does not meet these requirements, the field and space-time dynamics may eventually make the field leave the slow-roll regime everywhere. In Fourier space, this can alternatively be seen as due to nonlinear mixing between super-Hubble and sub-Hubble modes in the field and Einstein equations.

These problems are generic for scalar potentials where inflation occurs only at a scale $V_{0} \ll M_{P}^{4}$ so that, to make matters worse, inflation can only be triggered long after the Planck era. For plateau-like potentials inflation thus seems to require finely-tuned initial conditions for the field value, its velocity and degree of homogeneity, and also for the initial spatial curvature of the Universe following the Planck era. Some authors have even gone as far as saying that the inflationary paradigm is in considerable risk of failing [12] (see, however, Refs. [21, 22] for a different point of view).

Historically, considerations of the early universe have either treated the cosmological dynamics in the thermal limit, such as during the hot Big Bang regime, or in a zero temperature state, such as during inflation. The proposal of the warm inflation paradigm [23-25] showed that the dynamics slightly perturbed away from the thermal limit can have important consequences with robust consistency with data. In particular, Refs. [26, 27] have shown that going beyond the conventional setup for inflation and including interactions between the inflation and other degrees of freedom generically lowers the tensor-to-scalar ratio in monomial models, rendering them into agreement with observations. These interactions, which must always be present to ensure the transition from inflation to a radiation-dominated Universe, may lead to finite-temperature fluctuation-dissipation effects that can (depending on the model building details) potentially modify the dynamics of the inflaton field, at the level of both the homogeneous background value and the small perturbations about the latter. These effects are associated with particle production and, in the warm inflation scenario, are themselves capable of sustaining a nearly-thermal bath during the inflationary expansion.

More generally, there are several periods during the evolution of the early Universe where the Universe is slightly away from equilibrium and fluctuation-dissipation dynamics could be present. Such regimes may be essential for explaining important features of the observed 
Universe. In particular it has been shown that fluctuation-dissipation dynamics can play a relevant role in cosmic phase transitions [28] and baryogenesis [29] (for other applications of the effects of noise and dissipation in cosmology, see, for example, Refs. [30-36]).

The question that naturally arises from these previous studies is whether fluctuationdissipation effects can play any role in the pre-inflationary era and, in particular, whether they offer any novel insight on the problem of initial conditions, in particular for the plateaulike potentials for which this problem is most severe as discussed above. The presence of a friction term in the inflaton's equation of motion has already been shown to have a substantial impact in determining the minimum size of the initial inflationary patch [37]. In Ref. [37], it has been argued that if before inflation strong dissipation (characterized by a dissipation coefficient $\Upsilon$ compared to the Hubble expansion rate $H$ satisfying $\Upsilon>H$ ) is present, then it will damp fluctuations of modes with physical wavenumber $k<\Upsilon$. This means smoothness of the initial patch size need not be required at the Hubble scale $1 / H$ but over the much smaller scale $1 / \Upsilon$. For scales bigger than that, since dissipation will damp the modes, it will prevent nonlinear dynamics from becoming significant. The works in Ref. [14] were amongst the first to discuss the importance of an initial thermal state for the problem of initial conditions in "new inflation" models with a plateau-like potential. These previous works captured much of the important physics of the problem but were based in several ways on intuitive reasoning. In the present paper, we will show that fluctuation-dissipation dynamics during a radiation-dominated pre-inflationary epoch can indeed lead to such a thermalized state, where the homogeneous inflaton value naturally becomes localized within a flat plateau about the origin, thus setting the necessary conditions for the onset of inflation (see e.g. [38] for previous discussions of the role of the pre-inflationary dynamics in setting initial conditions for inflation).

While the framework of fluctuation-dissipation dynamics allows us to derive the key results in Ref. [14], we will go further and discuss in detail the conditions under which such a thermalized state can consistently be reached without spoiling the inflationary dynamics nor its observational predictions. This, of course, requires significant interactions between the inflaton and other degrees of freedom in the pre-inflationary thermal bath, which is widely believed to be incompatible with successful inflationary models (see, e.g., Ref. [39] for an earlier account on this). We wish, in particular, to challenge this prevailing view, and discuss concrete scenarios where a successful field localization on the plateau may be achieved.

In addition, we will show that thermal effects may keep playing an important role even after the inflaton's potential energy becomes dominant, since for a flat plateau about the origin the field's mass is dominated by thermal corrections until the temperature drops below the mass of the thermalized particles with which it interacts. This leads to a thermal inflation period that follows the early radiation era but precedes the final slow-roll regime. By lowering the temperature at which the field starts to move away from the origin, this period also suppresses nonlinear interactions between the homogeneous field component and its thermalized sub-Hubble modes, such that field inhomogeneities play a much less important role than previously estimated.

Finally, we will discuss the possibility of two periods of inflation occurring in the early Universe. A first period of chaotic inflation along a monomial branch of the potential can be triggered already at the Planck scale as discussed above. The energy stored in the inflaton field at the end of this period still exceeds largely the height of the plateau, so it is possible that after reheating the inflaton becomes localized about the origin due to fluctuation-dissipation effects and a second period of slow-roll eventually begins. The final 50-60 e-folds of inflation 
will then occur along the plateau-like region, thus combining the attractive features of both chaotic and new inflation within the same framework ${ }^{2}$.

This paper is organized as follows. In Sec. 2 we give the Langevin dynamics describing the evolution of the inflaton coupled to fermionic fields in the pre-inflationary thermal bath, when the latter dominate the energy balance. We explicitly verify that super-Hubble field modes become localized at the origin if their decay rate comes to exceed the expansion rate. In Sec. 3 we analyze the conditions under which this localization can be achieved, discussing in particular the compatibility of significant interactions between the inflaton and other degrees of freedom and quantum corrections in the effective potential, as well as the role of sub-Hubble modes and the initial conditions following the Planck era. In Sec. 4, we apply our results to a concrete example of a plateau-like scalar potential of the symmetry breaking form, and show explicitly that a period of thermal inflation may follow the early radiation era. We also discuss the possibility of an early period of chaotic inflation and the subsequent role of fluctuation-dissipation dynamics. Our main conclusions are summarized in Sec. 5 and we include two appendices with additional technical details on the dynamics and predictions of inflation for the concrete example considered and for a Higgs-like potential.

\section{Dynamical evolution of the inflaton field coupled to a radiation bath}

We start by describing the evolution of the inflaton field in a pre-inflationary era dominated by a radiation bath with which the inflaton interacts. We will describe the radiation bath in terms of a set of $N_{F}$ fermion species coupled to the inflaton through a standard Yukawa coupling of the form:

$$
\mathcal{L}_{Y}=-g \phi \sum_{i=1}^{N_{F}} \bar{\psi}_{i} \psi_{i} .
$$

The fermions acquire mass through the coupling to the inflaton and also possibly through other interactions within the thermal bath. For example, when interacting with abelian gauge bosons with strength $e$, which keep the fermions in thermal equilibrium [43] at a temperature $T$, the fermions can acquire a thermal plasma mass $m_{\psi}^{2}=e^{2} T^{2} / 8$. We will assume that these masses are negligible with respect to the temperature, $m_{\psi} \ll T$, but still larger than the Hubble parameter, $m_{\psi}>H$, such that a flat spacetime quantum field theory calculation can be used when evaluating the decay width of the inflaton into fermions. We will also neglect the tree-level inflaton mass, assuming that the field values of interest correspond to a region of the scalar potential with negligible curvature compared to the ambient temperature. These approximations will need to be checked a posteriori for consistency of the calculation. This is done when we present an explicit example in Sec. 4.

The inflaton nevertheless acquires a thermal mass through the coupling to the fermions in the radiation bath, which to leading order is given by [43]

$$
m_{\phi}^{2}=\alpha^{2} T^{2}, \quad \alpha^{2}=\frac{g^{2} N_{F}}{6} .
$$

Interactions with the thermal bath also leads to fluctuation-dissipation effects. For $m_{\phi} \gtrsim H$, all inflaton Fourier modes are oscillating, as we will see below, and the relevant dissipation

\footnotetext{
${ }^{2}$ For similar ideas using an early inflationary epoch that can precede a second inflation along a plateau, which could ease the initial conditions problem on the latter, see, e.g., Refs. [40-42]. However note that among other differences, none of these works considered the importance of an intermediary reheating phase.
} 
coefficient corresponds to the on-shell decay width into fermions [44], while decay into bosons (either scalar or gauge) particles can be neglected ${ }^{3}$. At finite temperature, for modes of physical three-momentum $p$, the inflaton decay width is given by [45]

$$
\Gamma_{\phi}(p)=\frac{3 \alpha^{2} m_{\phi}^{2}}{4 \pi \omega_{p}}\left[1+\frac{2 T}{p} \ln \left(\frac{1+e^{-\omega_{+} / T}}{1+e^{-\omega_{-} / T}}\right)\right],
$$

where $\omega_{p}=\sqrt{p^{2}+m_{\phi}^{2}}, \omega_{ \pm}=\left|\omega_{p} \pm p\right| / 2$ and $p=|\mathbf{p}|$ is the modulus of the physical momentum. Since we will be mainly interested in low-momentum modes, $p \ll T$, and given that $m_{\phi} \lesssim T$ for perturbative couplings, we can approximate the decay width by

$$
\Gamma_{\phi}(p) \simeq \frac{3 \alpha^{2} m_{\phi}^{2}}{4 \pi \omega_{p}}\left[1-2 n_{F}\left(\frac{m_{\phi}}{2}\right)\right] \simeq \frac{3 \alpha^{2} m_{\phi}^{3}}{16 \pi \omega_{p} T}
$$

where $n_{F}$ is the Fermi-Dirac distribution function. We point out that the additional factor $m_{\phi} / T<1$ in the finite temperature decay width with respect to the zero-temperature result is due to Pauli blocking and reflects the phase space decrease at high temperatures, due to the Fermi-Dirac distribution in eq. (2.4). However, since the inflaton mass $m_{\phi}$ increases with temperature, the decay width is, in fact, larger than at $T=0$. Note that, in a radiationdominated universe, we have

$$
a(t)=\left(t / t_{i}\right)^{1 / 2}, \quad H=\frac{1}{2 t}, \quad T(t)=T_{i}\left(t / t_{i}\right)^{-1 / 2},
$$

where we set $a\left(t_{i}\right)=1$ and the initial time is determined by the Hubble parameter when the initial conditions for the total energy density are set. This implies that $m_{\phi} \propto t^{-1 / 2}$ and since $p=k / a$ for comoving momentum $k$, we also have $\omega_{k} \equiv \omega_{p} \propto t^{-1 / 2}$. This yields $\Gamma_{\phi} \propto 1 / \sqrt{t}$, which means that the conditions $\Gamma_{\phi}, m_{\phi} \gtrsim H$ will necessarily be satisfied given a sufficiently long time. For a radiation-dominated universe, the Friedmann relation becomes

$$
H=\sqrt{\frac{\pi^{2}}{90}} g_{*}^{1 / 2} \frac{T^{2}}{M_{P}},
$$

where $g_{*}$ denotes the number of relativistic degrees of freedom in the thermal bath, which includes the fermions and in general other species not directly coupled to the inflaton and $M_{P}$ is the reduced Planck mass, $M_{P}=2.435 \times 10^{18} \mathrm{GeV}$. Thus, we have in the limit of vanishing momentum that

$$
\frac{\Gamma_{\phi}}{H} \simeq 0.18 \frac{\alpha^{4}}{g_{*}^{1 / 2}} \frac{M_{P}}{T},
$$

\footnotetext{
${ }^{3}$ Note that e.g. for an interaction with scalar bosons of the form $g^{2} \phi^{2} \chi^{2}$, the trilinear vertex leading to the $\phi \rightarrow \chi \chi$ decay is proportional to the tree-level scalar mass $g|\phi|<T$ for relativistic bosons, and this results in a suppression of the bosonic decay channels relative to the fermionic ones by a factor $(g \phi / \alpha T)^{2} \ll 1$. In any case, the inclusion of additional decay channels will not change our conclusions significantly and in fact it will only enhance the localization effect described below. Additional non-perturbative effects that we are not taking into account in this analysis may also enhance this effect. The same is true if scattering processes are also included. For instance, the scattering processes $\psi_{i} \bar{\psi}_{i} \leftrightarrow \phi \phi$ and $\psi_{i} \phi \leftrightarrow \psi_{i} \phi$ correspond to higher-loop contributions to the imaginary part of the inflaton's self-energy. They are thus suppressed compared to the decays considered in this work, which correspond to one-loop processes, and their contribution to the damping rate is further suppressed in the large $N_{F}$ limit for finite $\alpha$.
} 
which implies that dissipative effects will become significant for temperatures parametrically below the Planck scale. Since $\Gamma_{\phi} \lesssim m_{\phi}$ for perturbative couplings, the inflaton field will begin oscillating before dissipative effects become significant.

Assuming a flat FRW geometry, each Fourier mode of the inflaton field will satisfy the Langevin-like equation of the form $[46,47]$

$$
\ddot{\phi}_{k}+\left(3 H+\Gamma_{\phi}(k)\right) \dot{\phi}_{k}+\omega_{k}^{2} \phi_{k}=\xi_{k},
$$

where $\xi_{k}$ is a stochastic noise term that encodes the backreaction of the thermal bath on the evolution of the field and is related to the dissipation coefficient through the fluctuationdissipation relation $[46,47]$

$$
\left\langle\xi_{k}\left(t_{1}\right) \xi_{k^{\prime}}\left(t_{2}\right)\right\rangle=2 \Gamma_{\phi}(k) T \frac{(2 \pi)^{3}}{a^{3}} \delta^{3}\left(\mathbf{k}+\mathbf{k}^{\prime}\right) \delta\left(t_{1}-t_{2}\right) .
$$

This relation is valid in the high-temperature regime, when the fields coupled to the inflaton are relativistic, and the noise term is approximately white and Gaussian (with zero average).

To determine the evolution of the Fourier modes, we assume a narrow decay width $\Gamma_{\phi} \ll \omega_{k}$ and large frequency $\omega_{k} \gg H$, which should both be valid when the couplings are small and $T \gg H$. The equation can be put into a suitable form for a JWKB analysis by introducing $\tilde{\phi}_{k}$ from

$$
\phi_{k}=a^{-3 / 2} e^{-\Gamma_{\phi} /(2 H)} \tilde{\phi}_{k} .
$$

The approximations mentioned above now imply that

$$
\ddot{\tilde{\phi}}_{k}+\omega_{k}^{2} \tilde{\phi}_{k} \approx \tilde{\xi}_{k}=a^{3 / 2} e^{\Gamma_{\phi} /(2 H)} \xi_{k} .
$$

The JWKB solutions to the homogeneous equation are

$$
\tilde{\phi}_{k}^{ \pm} \approx \omega_{k}^{-1 / 2} e^{ \pm i \int \omega_{k} d t} .
$$

The solutions (2.10) then yield oscillating field modes, the amplitude of which redshifts with expansion and also decays exponentially in time due to dissipative effects. As seen above, the latter will become significant at parametrically low temperatures and we would expect the field to completely decay away given enough time. This is analogous to the damping of a Brownian particle's motion in a molecular gas due to the average friction effect resulting from random collisions with the molecules in the environment [48].

The JWKB solutions provide the Green functions which we can use to solve the inhomogeneous equation,

$$
\tilde{\phi}_{k}(t)=\int_{t_{i}}^{t} \tilde{G}(t, s) \tilde{\xi}_{k}(s)
$$

where

$$
\tilde{G}(t, s) \approx \omega_{k}(t)^{-1 / 2} \omega_{k}(s)^{-1 / 2} \sin \int_{s}^{t} \omega_{k}\left(t^{\prime}\right) d t^{\prime} .
$$

The quantity of interest is the power spectrum of fluctuations,

$$
\left\langle\tilde{\phi}_{k}(t) \tilde{\phi}_{k^{\prime}}(t)\right\rangle=\int_{t_{i}}^{t} d s_{1} \int_{t_{i}}^{t} d s_{2} \tilde{G}\left(t, s_{1}\right) \tilde{G}\left(t, s_{2}\right)\left\langle\tilde{\xi}_{k}\left(s_{1}\right) \tilde{\xi}_{k}\left(s_{2}\right)\right\rangle .
$$


Using the noise correlation relation Eq. (2.9), and restoring the original field modes, we may write the power spectrum as

$$
\left\langle\phi_{k}(t) \phi_{k^{\prime}}(t)\right\rangle=2 e^{-\Gamma_{\phi} / H(t)} a(t)^{-3} \int_{t_{i}}^{t} \frac{d s}{\omega_{k}(s) \omega_{k}(t)}\left[\sin ^{2} \int_{s}^{t} \omega_{k}\left(t^{\prime}\right) d t^{\prime}\right] \Gamma_{\phi}(s) T(s) e^{\Gamma_{\phi} / H(s)} .
$$

Now a remarkable simplification occurs for the dissipation term given above. We notice that $\Gamma_{\phi} / H \propto a$, and consequently

$$
\frac{\Gamma_{\phi} T}{\omega_{k}} d s \propto d a .
$$

The equal-time correlator can then be obtained by averaging over the field oscillations (using that $\left\langle\sin ^{2} x\right\rangle=1 / 2$ ) and integrating a simple exponential to give

$$
\begin{aligned}
\left\langle\phi_{k}(t) \phi_{k^{\prime}}(t)\right\rangle & \simeq \frac{T(t)}{\omega_{k}^{2}(t) a^{3}(t)}\left[1-e^{-\left[\Gamma_{\phi} / H(t)-\Gamma_{\phi} / H\left(t_{i}\right)\right]}\right](2 \pi)^{3} \delta^{3}\left(\mathbf{k}+\mathbf{k}^{\prime}\right) \\
& \simeq \frac{T(t)}{\omega_{k}^{2}(t) a^{3}(t)}(2 \pi)^{3} \delta^{3}\left(\mathbf{k}+\mathbf{k}^{\prime}\right),
\end{aligned}
$$

where we have taken $\Gamma_{\phi} \gtrsim H$ in the second equality.

It is important to point out that the result (2.18) applies not only to sub- but also to super-Hubbles modes in the present set up. A priori one should expect super-Hubble modes not to be thermal. They should be frozen and maybe squeezed, as super-Hubble modes of cosmological perturbations are [49]. However, we are considering the radiation epoch, and in this epoch super-Hubble fluctuations are not frozen out and are not squeezed. The reason is that the usual squeezing factor $a^{\prime \prime} / a$ vanishes. In addition, we are considering a massive field, and so the super-Hubble modes will be oscillating and their evolution will remain adiabatic. In this way, the fluctuation term conveys thermal equilibrium amplitude values to super-Hubble modes.

Note that the power spectrum redshifts as $t^{-1}$ in the radiation dominated era, which is the regime we assume to be most relevant for the pre-inflationary era. The result still applies, however, when radiation and potential energy terms are comparable, and we shall need this later. Note also that the field variance becomes independent of the friction coefficient for $\Gamma_{\phi} \gtrsim H$. Thus, the noise term counteracts the effects of dissipation, making the field modes evolve towards an equilibrium configuration determined by the ambient temperature $T(t)$. This would follow from local flatness and the equivalence principle for scales smaller than the horizon (see, e.g., Ref. [34]), but the important point here is that the result holds on long as well as short scales. Again, this is analogous to the motion of a Brownian particle described by the Langevin equation, where the random effect of molecular collisions does not really lead to the complete damping of the particle's motion through dissipative friction but rather to thermal fluctuations of the particle's velocity in an equilibrium configuration [48].

We may now use the result given by Eq. (2.18) to determine the average field value in configuration space. We are mainly interested in the "classical" or "homogeneous" field component, since only the low-momentum or superhorizon modes, $k<a H$, can lead to a slowroll inflationary regime, while sub-horizon modes will redshift away more quickly $[15,50,51]$. Since the field fluctuations in a thermal bath are classical in nature, we may use the Fourier mode decomposition for a real classical field,

$$
\phi(\mathbf{x}, t)=\int \frac{d^{3} k}{(2 \pi)^{3}} \phi_{k}(t) \cos \left(\mathbf{k} \cdot \mathbf{x}+\alpha_{\mathbf{k}}\right),
$$


where $\alpha_{\mathbf{k}}$ are mode-dependent phases. Assuming the latter to be randomly distributed and thus averaging to zero, we may then write the average field value on superhorizon scales as:

$$
\begin{aligned}
\left\langle\phi_{c}^{2}(\mathbf{x}, t)\right\rangle & =\int_{k, k^{\prime}<a H} \frac{d^{3} k}{(2 \pi)^{3}} \frac{d^{3} k^{\prime}}{(2 \pi)^{3}}\left\langle\phi_{k}(t) \phi_{k^{\prime}}(t)\right\rangle \cos (\mathbf{k} \cdot \mathbf{x}) \cos \left(\mathbf{k}^{\prime} \cdot \mathbf{x}\right) \\
& \simeq \frac{T}{4 \pi^{2} a^{3}} \int_{0}^{a H} \frac{k^{2} d k}{\omega_{k}^{2}} \int_{-1}^{1} d \cos \theta \cos ^{2}(k r \cos \theta) \\
& \simeq \frac{T}{4 \pi^{2} a^{3}} \int_{0}^{a H} \frac{k^{2} d k}{\omega_{k}^{2}}\left[1+\frac{\sin (2 k r)}{2 k r}\right] .
\end{aligned}
$$

The oscillatory term between brackets in the last line in Eq. (2.20) makes the exact integration rather cumbersome, but since it is roughly between 1-2, we may obtain a good estimate for the field variance by replacing it with an $\mathcal{O}(1)$ number, say 2 for concreteness and to give the largest possible variance. This then yields the result

$$
\left\langle\phi_{c}^{2}\right\rangle \sim \frac{T}{2 \pi^{2} a^{3}} \int_{0}^{a H} \frac{k^{2} d k}{\omega_{k}^{2}} \sim \frac{H^{3} T}{6 \pi^{2} m_{\phi}^{2}} \sim \frac{1}{6 \pi^{2} \alpha^{2}} \frac{H^{3}}{T},
$$

where we have used that $m_{\phi} \gtrsim H$ in the pre-inflationary era. This implies that the meansquare classical field fluctuation decreases as $t^{-5 / 2}$ and we may write it only in terms of the temperature of the thermal bath to finally obtain the result

$$
\left\langle\phi_{c}^{2}\right\rangle \sim \frac{\pi}{6 \alpha^{2}}\left(\frac{g_{*}}{90}\right)^{3 / 2} \frac{T^{5}}{M_{P}^{3}} .
$$

From the results given by Eqs. (2.21) and (2.22), we thus conclude that, asymptotically, the average inflaton field variation is below the ambient temperature and also below the Hubble scale since $H^{3} / T<H^{2}$. This holds when the effective coupling is not too small, but notice that we required $m_{\phi} \simeq \alpha T \gtrsim H$ for consistency of the calculation.

\section{General Consistency Conditions Analysis}

The analysis given in the previous section shows that fluctuation-dissipation effects can localize the homogeneous inflaton field at the origin with a parametrically small dispersion $\Delta \phi_{c} / H \sim 0.1 \alpha^{-1}(H / T)^{1 / 2}$, as inferred from the result in Eq. (2.21). This will occur in a preinflationary radiation-dominated epoch provided that the inflaton field interacts significantly with the ambient radiation, modeled by a set of fermion species in our analysis. We are interested in scalar potentials that have a flat plateau about the origin, where the curvature is negligible and the inflaton mass is dominated by thermal effects. We will consider a specific example of such a potential in Sec. 4, but in general we may consider a flat plateau region of height $V_{0}$. Thus, when the field is localized in this plateau, the inflaton potential will dominate over the energy density in radiation at temperatures below the equality temperature $T_{e q}$. This can be obtained by equating $\rho_{R}\left(T_{e q}\right)=V_{0}$, which yields:

$$
T_{e q}=\left(\frac{30}{\pi^{2}}\right)^{1 / 4} g_{*}^{-1 / 4} V_{0}^{1 / 4}
$$

We may relate the height of the plateau with the tensor-to-scalar ratio and the known amplitude of scalar curvature perturbations, $\Delta_{\mathcal{R}}^{2} \simeq 2.2 \times 10^{-9}$ [4], generated in the slow-roll 
regime,

$$
r=\frac{2}{3 \pi^{2}} \Delta_{\mathcal{R}}^{-2} \frac{V_{0}}{M_{P}^{4}}
$$

Using Eq. (2.7), one may then obtain a lower bound on the effective coupling $\alpha$, defined in Eq. (2.2), which is required such that $\Gamma_{\phi}>H$ before the transition temperature for vacuum dominance over radiation is reached and the pre-inflationary radiation era comes to an end. This yields

$$
\alpha \gtrsim 0.24 g_{*}^{1 / 16}\left(\frac{r}{10^{-6}}\right)^{1 / 16} .
$$

Hence, the coupling between the inflaton and the thermal bath has to be quite large unless inflation occurs at very low scales and yields a negligible tensor-to-scalar ratio. Otherwise, dissipative friction will not have enough time to damp any initially large field values and stabilize the variance at the value computed in the previous section. Such coupling values may be too large to yield a successful inflationary model, since radiative corrections to the scalar potential may spoil its flatness and fail to give the observed amplitude of the primordial curvature perturbation spectrum. This requires further examination of the effective potential during inflation. Likewise, we have to make sure that the energy density in the inhomogeneous (sub-Hubble) inflaton modes remains sub-dominant, so as not to spoil the onset of accelerated expansion. Finally, we also need to ensure that, as assumed, the fermions remain light throughout the whole of the pre-inflationary era, which is crucial for the thermalization and consequent localization of the inflaton field. Below we analyze each of these issues in more detail.

\subsection{Radiative Corrections and the Flatness of the Inflaton Potential}

As shown above, the coupling of the inflaton to the radiation bath fields, Eq. (3.3), has to be rather large so as to allow a sufficiently fast localization of the inflaton field with small variance. This in turn can potentially lead to large radiative corrections to the inflaton potential, possibly endangering its flatness. Two possible solutions to this problem, and that we discuss next, can be given in terms of inflation models based on supersymmetry, or, alternatively, by the appropriate application of the renormalization procedure determining these radiative corrections.

In supersymmetric models of inflation, large (although perturbative) couplings may be allowed. Despite supersymmetry being broken by the finite energy density during inflation, there is still a partial cancellation of bosonic and fermionic radiative corrections. For example, for a superpotential of the form $W=g \Phi X_{i}^{2} / 2+f(\Phi)$, which gives a Yukawa term of the form in Eq. (2.1), if the inflaton is the scalar component of the $\Phi$ supermultiplet and the fermions are part of the chiral multiplets $X_{i}, i=1, \ldots, N_{F}$, one obtains the one-loop correction to the scalar potential [52]:

$$
\Delta V_{S U S Y}^{(1)}(\phi)=\frac{3 \alpha^{2}}{8 \pi^{2}} \ln \left(\frac{g^{2} \phi^{2}}{\mu^{2}}\right) V(\phi)
$$

where $\mu$ is the renormalization scale in the $\overline{\mathrm{MS}}$ scheme, and $V(\phi)=\left|f^{\prime}(\phi)\right|^{2}$ is the tree-level scalar potential, such that one-loop corrections give naturally a sub-dominant contribution to the effective potential and do not significantly modify the tree-level predictions. Note that 
we may easily construct a supersymmetric version of the analysis performed in the previous section, with bosonic fields contributing to the inflaton's thermal mass and decay width. The contribution of bosons to the latter is, in fact, suppressed at high temperatures as we have already discussed, while the thermal mass contribution is analogous to that of the fermions up to numerical factors. Supersymmetry can then allow for the significant coupling between the inflaton and a radiation bath required for the localization of its homogeneous modes close to the origin without considerably changing the form of the potential at zero-temperature.

Alternatively, it has also been argued that, if the particles coupled to the inflaton are heavy compared to the relevant energy scale $\mu$ during inflation, their contribution to the effective potential is further suppressed, in the spirit of the decoupling theorem [53]. This becomes explicit using a mass-dependent renormalization scheme [54], whereas in mass-independent schemes, such as the modified minimal subtraction $\overline{\mathrm{MS}}$ scheme used in dimensional regularization, it has to be enforced by hand. In particular, in a mass-dependent renormalization scheme, the one-loop contribution of fields coupled to the inflaton is given, at one-loop order, by

$$
\Delta V_{M D}^{(1)}(\phi)=\frac{g^{4} N_{F} \phi^{4}}{64 \pi^{2}}\left[\ln \left(\frac{M^{2}}{\mu^{2}}\right)-I\left(\frac{M^{2}}{\mu^{2}}\right)\right],
$$

where the function $I(a)=\ln (a)-2-\sqrt{1+4 a} \ln [(\sqrt{1+4 a}-1) /(\sqrt{1+4 a}+1)]$ effectively replaces the $3 / 2$ factor that one obtains in the $\overline{\mathrm{MS}}$ regularization scheme. This has been derived in Ref. [54] for a scalar interaction $g^{2} \phi^{2} \chi^{2} / 2$, yielding an effective mass squared for the $\chi$ particles given by $M^{2}=m_{\chi}^{2}+g^{2} \phi^{2}$. An analogous result can also be derived for fermions, which gives an expression of the form of Eq. (3.5), but with an opposite sign. For heavy fields, with $\mu \ll M$, Eq. (3.5) becomes

$$
\Delta V_{M D}^{(1)}(\phi) \simeq-\frac{g^{4} N_{F} \phi^{4}}{384 \pi^{2}} \frac{\mu^{2}}{M^{2}} .
$$

In particular, if the field-independent mass in $M^{2}=m_{\chi}^{2}+g^{2} \phi^{2}$ can be neglected, we immediately see that this correction to the potential is quadratic in $\phi$, thus shifting its squared mass by an amount $\left(\alpha^{2} / 32 \pi^{2}\right) \mu^{2}$. Arguably the relevant energy scale during inflation is the Hubble scale, $\mu \sim H_{\text {inf }}$, such that this correction to the inflaton mass is small even for the relatively large values of the effective coupling $\alpha$ required for thermalization, and does not spoil the flatness of the potential. Note that the temperature during the pre-inflationary radiation era $T>H>H_{\text {inf }}$, so that we may have the hierarchy $T>M>H_{\text {inf }}$ for which fields in the thermal bath are relativistic before inflation but decouple during the slow-roll phase. The appropriate choice for the renormalization scale during inflation remains, however, the subject of some discussion in the literature, but it is nevertheless clear that there are possible scenarios where the inflaton can couple sufficiently strongly to a pre-inflationary thermal bath without spoiling the required properties of the scalar potential at the quantum level.

\subsection{Kinetic and gradient energies before inflation}

We have concluded in the previous section that the super-Hubble (or homogeneous) inflaton modes become localized at the origin due to fluctuation-dissipation effects. We need, however, to make sure that (i) their velocity and spatial variation are also kept small and (ii) the energy density stored in higher-momentum modes is diluted fast enough compared to the potential energy. These conditions are crucial for the homogeneous field to enter a slow-roll regime. 
As we have seen earlier in the previous Section 2, provided that $\Gamma_{\phi}>H$, the inflaton field coupled to the radiation bath will tend to an equilibrium solution characterized by a "classical" component of average $\left\langle\phi_{c}^{2}\right\rangle \sim H^{3} T / 6 \pi^{2} m_{\phi}^{2}$, with $m_{\phi}^{2} \simeq \alpha^{2} T^{2}$. From the inhomogeneous solution we have obtained in that Section, we may also compute the average field velocity and gradients, in order to better understand whether the field localization achieved by interactions with the thermal bath leads to appropriate initial conditions for inflation with a plateau-like potential.

First, differentiating Eq. (2.13) with respect to time, again using the JWKB approximation, we obtain:

$$
\dot{\tilde{\phi}}_{k} \approx \omega_{k}(t) \int_{t_{i}}^{t}\left[\int_{s}^{t} \cos \omega_{k}\left(t^{\prime}\right) d t^{\prime}\right] \tilde{\xi}_{k}(s) \frac{d s}{\sqrt{\omega_{k}(s) \omega_{k}(t)}}
$$

We may then proceed as for the computation of the field variance to obtain the variance of the field velocity. In this case the main differences are the factors outside the integral and the fact that the integral includes a cosine function rather than a sine. Since we are averaging over field oscillations, the latter difference is irrelevant and it is straightforward to obtain after some algebra the result

$$
\left\langle\dot{\phi}_{k}(t) \dot{\phi}_{k^{\prime}}(t)\right\rangle \simeq \frac{T(t)}{a^{3}(t)}(2 \pi)^{3} \delta^{3}\left(\mathbf{k}+\mathbf{k}^{\prime}\right)=\omega_{k}^{2}(t)\left\langle\phi_{k}(t) \phi_{k^{\prime}}(t)\right\rangle .
$$

The above result is what one would expect for an oscillating field with frequency $\omega_{k}$. For small momentum modes such that $p=k / a \ll m_{\phi} \sim \alpha T$, this frequency is simply the field's thermal mass, such that it is easy to obtain the average squared field velocity for the classical component, including all comoving momenta up to $k=a H$,

$$
\left\langle\dot{\phi}_{c}^{2}\right\rangle \simeq m_{\phi}^{2}\left\langle\phi_{c}^{2}\right\rangle \simeq \frac{H^{3} T}{6 \pi^{2}} .
$$

This implies that the average kinetic energy in the classical field decays as $a^{-7}$ during the pre-inflationary radiation era, and so much faster than the radiation fluid itself.

The above calculation also provides us with the initial conditions in phase space for the classical (homogeneous) field at the equality temperature, given in Eq. (3.1). This yields

$$
\begin{gathered}
\phi_{c} \sim \sqrt{\left\langle\phi_{c}^{2}\right\rangle} \simeq 0.08 \frac{g_{*}^{1 / 8}}{\alpha}\left(\frac{V_{0}}{M_{P}^{4}}\right)^{5 / 8} M_{P} \simeq 10^{-7} \frac{g_{*}^{1 / 8}}{\alpha}\left(\frac{r}{0.01}\right)^{5 / 8} M_{P}, \\
\phi_{c}^{\prime} \sim H \sqrt{\left\langle\dot{\phi}_{c}^{2}\right\rangle} \simeq 0.13 g_{*}^{-1 / 8}\left(\frac{V_{0}}{M_{P}^{4}}\right)^{3 / 8} M_{P} \simeq 4 \times 10^{-5} g_{*}^{-1 / 8}\left(\frac{r}{0.01}\right)^{3 / 8} M_{P} .
\end{gathered}
$$

Notice that this is still far from a slow-roll solution, since $\phi_{c}^{\prime} / \phi_{c} \sim \alpha T / H \gg 1$, which is naturally expected since the field is oscillating. We will nevertheless show in the next section, where we consider a concrete example of a plateau-like potential, that these initial conditions can yield a sufficiently long period of inflation in the slow-roll regime. Note also that the recent Planck results [4] yield $H_{\text {inf }} \lesssim 10^{13} \mathrm{GeV}$ for the Hubble parameter during inflation, such that $V_{0} \ll M_{P}^{4}$. Thus, both the average field value and the average field velocity are 
(very) sub-Planckian. Note, in addition, that the average super-horizon field variance can be written as

$$
\left\langle\phi_{c}^{2}\right\rangle \simeq \frac{2}{3 \alpha^{2}}\left(\frac{H}{T}\right)\left(\frac{H}{2 \pi}\right)^{2} .
$$

Thus, in the presence of the thermal bath the average field fluctuations on super-horizon scales are smaller than the quantum fluctuations in the de Sitter inflationary phase on the scale of the horizon with amplitude $\sim H / 2 \pi$.

We may proceed analogously to compute the gradient energy, noting that

$$
\begin{aligned}
\left\langle(\nabla \phi(\mathbf{x}, t))^{2}\right\rangle & =\int \frac{d^{3} k}{(2 \pi)^{3}} \int \frac{d^{3} k^{\prime}}{(2 \pi)^{3}} \mathbf{k} \cdot \mathbf{k}^{\prime}\left\langle\phi_{k}(t) \phi_{k^{\prime}}(t)\right\rangle \sin (\mathbf{k} \cdot \mathbf{x}) \sin \left(\mathbf{k}^{\prime} \cdot \mathbf{x}\right) \\
& =\frac{T}{a^{3}} \int \frac{d^{3} k}{(2 \pi)^{3}} \frac{k^{2}}{\omega_{k}^{2}} \sin ^{2}(\mathbf{k} \cdot \mathbf{x}) \\
& =\frac{T}{4 \pi^{2} a^{3}} \int \frac{k^{4} d k}{\omega_{k}^{2}}\left[1-\frac{\sin (2 k r)}{2 k r}\right]
\end{aligned}
$$

where in the last line we have performed the integral over the angular variables. The term within square brackets is $\mathcal{O}(1)$ and we may replace it by unity to estimate the gradient energy. For the classical field component, we include only the super-horizon modes in the momentum integral, which then gives for the gradient energy the result

$$
\frac{1}{2 a^{2}}\left\langle\left(\nabla \phi_{c}\right)^{2}\right\rangle \simeq \frac{1}{40 \pi^{2} \alpha^{2}} \frac{H^{5}}{T},
$$

which decays as $a^{-11}$. Note that this is essentially $H^{2}\left\langle\phi_{c}^{2}\right\rangle$ up to numerical factors. Hence, $\left\langle\phi_{c}^{2}\right\rangle$ gives the mean variation of the field over super-horizon distances.

In the calculations shown above we have considered only the classical field component, including only super-horizon momentum modes, since these are the only ones that may eventually enter a slow-roll regime after the radiation-dominated period has ended. It is nevertheless relevant to compute the average values of the different energy components when including higher-momentum modes. Our analysis is valid up to physical momenta of the order of the ambient temperature, $p=k / a \lesssim T$, for which $\omega_{k} \sim \alpha T$, for $\alpha \lesssim 1$. Highermomentum modes will naturally be (Boltzmann) suppressed in the thermal bath, such that we may safely neglect them. Thus, extending our earlier calculations to $k<a T$, we obtain that the average potential $\langle V\rangle$, field velocity energy density $\left\langle\rho_{\dot{\phi}}\right\rangle$ and the energy density in the field gradient $\left\langle\rho_{\nabla}\right\rangle$ are given, respectively, by

$$
\begin{aligned}
\langle V\rangle & =\frac{1}{2} m_{\phi}^{2}\left\langle\phi^{2}\right\rangle \simeq \frac{1}{4 \pi^{2}} T^{4}, \\
\left\langle\rho_{\dot{\phi}}\right\rangle & =\frac{1}{2}\left\langle\dot{\phi}^{2}\right\rangle \simeq \frac{1}{4 \pi^{2}} T^{4}, \\
\left\langle\rho_{\nabla}\right\rangle & =\frac{1}{2 a^{2}}\left\langle(\nabla \phi)^{2}\right\rangle \simeq \frac{1}{40 \pi^{2} \alpha^{2}} T^{4} .
\end{aligned}
$$

This shows that the potential, the kinetic and the gradient energies in sub-horizon modes are diluted like radiation. This should be expected, since for $\Gamma_{\phi}>H$ the inflaton field thermalizes, and its short-wavelength components should behave as relativistic degrees of freedom in 
the thermal bath. The numerical factors in Eqs. (3.15), (3.16) and (3.17) are not rigorous, due to the approximations considered when estimating them, but this shows that essentially the inflaton field can be split into two separate components: the long-wavelength modes, which may eventually enter the slow-roll regime; and the short-wavelength modes, which are essentially part of the thermal bath and should correspond to an additional relativistic degree of freedom that must be taken into account when computing $g_{*}$. Once the radiation energy density $\rho_{R}$ satisfies $\rho_{R}<V_{0}$, the long-wavelength modes will dominate the evolution if they enter a slow-roll regime, according to the discussion above, and neither the kinetic nor the gradient energies in the short-wavelength components can block inflation, since, like radiation, they will be diluted away.

Notice that one could worry that, even if the field's gradient energy is negligible, large fluctuations in the field could bring the classical component outside the plateau where slowroll is possible through nonlinear effects. The analysis above shows that the largest fluctuations of the field occur on sub-horizon scales of size $\sim T^{-1}$ and with amplitude $\sim T$. This would imply that the plateau of the inflaton potential must in general extend for $\Delta \phi \gtrsim T_{e q} \sim V_{0}^{1 / 4}$ about the origin, i.e., that the plateau's width must be at least comparable to its height. However, the inflaton's self-interactions are typically extremely weak and, as we will discuss in the next section with a concrete example, slow-roll does not start immediately at the equality temperature. We will thus leave the study of nonlinear mixing between sub- and super-Hubble modes to the next section.

\subsection{Thermalization and equipartition of energy}

Our analysis of the thermalization process prior to the inflationary regime relies on the assumption that the inflaton is coupled to thermalized light particles that dominate the energy balance before the onset of inflation. If this is the case, the inflaton field will be localized at the origin by thermal and fluctuation-dissipation effects as we have demonstrated, but we need to check the consistency of this assumption.

Since our analysis is based on general relativity and perturbative quantum field theory, it will only hold for energies below the Planck scale. We must then ask what are the reasonable initial conditions as the Universe emerges from the Planck era, when quantum gravity effects are presumably dominant. In principle, the Universe emerges from this era, i.e., quantum gravity effects become sub-leading, when the total energy density becomes sub-planckian, i.e., when $\rho_{T} \sim M_{P}^{4}$. Since gravity treats all species on equal footing, it is also reasonable to assume equipartition of energy after the Planck era. We assumed that there are $g_{*}$ relativistic degrees of freedom at this stage (and throughout the pre-inflationary stage) that quickly thermalize, and the inflaton field should thus carry as much energy density as each of these degrees of freedom. Thus, for $g_{*} \gg 1$, we have that

$$
\rho_{T} \sim g_{*} T^{4}+\rho_{\phi} \sim M_{P}^{4} \quad \Rightarrow \quad \rho_{\phi} \sim T^{4} \sim g_{*}^{-1} M_{P}^{4} .
$$

Hence, we expect that immediately after the Planck era the Universe will have a temperature parametrically below $M_{P}$ and the energy in the inflaton field is also parametrically subplanckian. Nevertheless, for a not too large number of relativistic degrees of freedom, we expect that at this stage $\rho_{\phi} \gg V_{0}$ and that the temperature is well above the equality temperature defined earlier, $T_{e q} \sim g_{*}^{-1 / 4} V_{0}^{1 / 4}$.

Consistency of the analysis requires, in particular, that the fermions are light, $g|\phi| \lesssim T$. If this is the case, our analysis shows that the field amplitude redshifts as $1 / a$ and is further 
reduced by fluctuation-dissipation effects through the inflaton decay into fermions. Since $T \propto 1 / a$, this implies that if the condition $g|\phi|<T$ is satisfied at the Planck era, it will be satisfied at all times afterwards. Thus, we need to check under which conditions one can have light fermions already at the Planck era, and analyze what happens otherwise.

To properly address the above consistency conditions for the localization of the inflaton field, it is more convenient to show how they work for an explicit example, as we will do in the following section.

\section{Initial Condition Problem for Plateau-Like Inflaton Potentials: An ex- plicit example}

The main constraints on any inflaton potential are that it must lead to enough inflation, with number of e-folds of expansion around or greater than $N_{e} \sim 50-60$, to give the correct amplitude of scalar curvature perturbations, $\Delta_{\mathcal{R}}^{2} \simeq 2.2 \times 10^{-9}$, and to produce satisfactory values for the observational quantities, like the tensor-to-scalar ratio $r$ and the spectral index $n_{s}$, which according to the recent Planck data [4] are constrained to the values $r<0.08(95 \%$ $\mathrm{CL}, \mathrm{TT}+$ lowP $+\mathrm{BKP})$ and $n_{s}=0.9655 \pm 0.0062(68 \% \mathrm{CL}$, Planck TT+lowP $)$, respectively.

The upper bound on the tensor-to-scalar ratio, in particular, implies that large-field cold inflation models, such as monomial potentials, are generically disfavored by the data, which thus seems to prefer scalar potentials with a plateau-like region. We are mainly interested in potentials for which the plateau is centered at $\phi=0$, in which case fluctuationdissipation effects may localize the field in the plateau during a pre-inflationary radiationdominated era as we have discussed in the previous sections. This suggests potentials with a $\mathbb{Z}_{2}$ reflection symmetry with minima displaced from the origin at $\phi= \pm v$. The simplest example of such a potential is, of course, the Higgs type of symmetry breaking potential $V(\phi)=\lambda\left(\phi^{2}-v^{2}\right)^{2} / 4$, but it is easy to check that such a potential only admits slow-roll solutions for $v \gtrsim M_{P}$. These solutions involve super-planckian field values, and whether this poses a problem for inflationary model-building has been the object of much discussion in the literature. Nevertheless, independently of this fact, when slow-roll solutions exist they are phase-space attractors, as we explicitly show in Appendix A. In this sense there is no real initial conditions problem for this renormalizable double-well potential, and it behaves very much like a large-field model, although it may lead to a tensor-to-scalar ratio within observational bounds.

A fine-tuning of initial conditions is only required in cases where slow-roll solutions are not phase-space attractors, and this occurs for plateau-potentials about the origin such that $V^{\prime}(0)=V^{\prime \prime}(0)=0$. This is the case of the Coleman-Weinberg potential $V=V_{0}\left[1+x^{4}\left(2 \ln x^{2}-1\right)\right]$, where $x=\phi / v$, that results from one-loop quantum corrections to a classically flat potential and, thus, yields the well-known radiative symmetry breaking mechanism. It is also the case of symmetry-breaking potentials with non-renormalizable terms, such as:

$$
V(\phi)=V_{0}\left(1-3 \frac{\phi^{4}}{v^{4}}+2 \frac{\phi^{6}}{v^{6}}\right)
$$

where $V_{0}=\lambda v^{4}$. We plot these two potentials in Fig. 1, where the similarities between them are apparent. In fact, they lead to quite similar observational predictions for $n_{s}$ and $r$ as can be easily checked.

For simplicity of the calculations, and despite its non-renormalizable nature, we will take the latter as the particular example of a plateau-like potential for which we will explicitly 


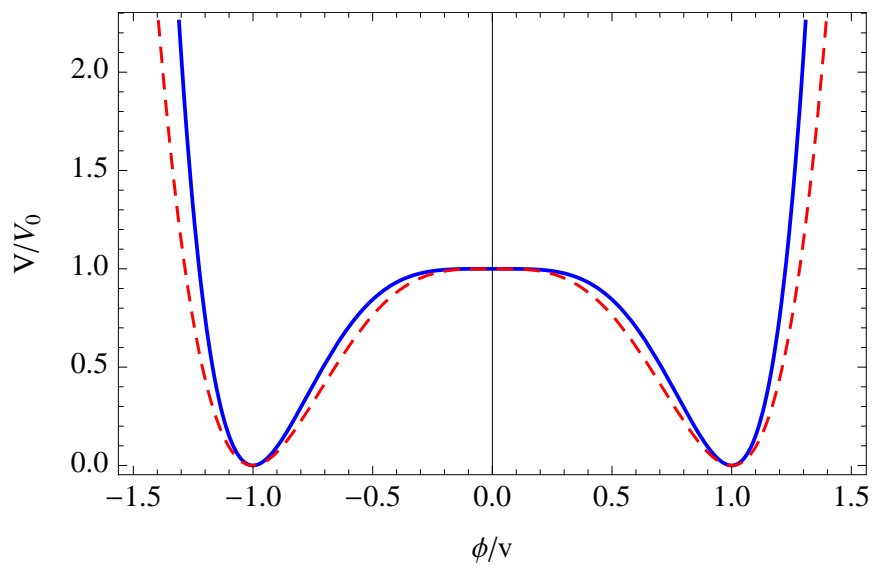

Figure 1. Non-renormalizable plateau-like potential (solid blue curve) and Coleman-Weinberg potential (dashed red curve).

check the efficiency and validity of the generic localization mechanism through fluctuationdissipation effects. The subsequent discussion will nevertheless apply in a similar way to the Coleman-Weinberg case and other similar plateau-like models such as the generic nonrenormalizable models described in Appendix B.

We note that with potentials of this form one can obtain (non-attractor) slow-roll solutions for sub-planckian field values, as explicitly shown in Appendix B. This leads to low values of the tensor-to-scalar ratio, possibly outside the reach of near-future experiments, and for the non-renormalizable potential in Eq. (4.1) one obtains in this case $n_{s} \simeq 0.94-0.95$. Although this is outside the window allowed by the latest Planck data, we point out that non-renormalizable plateau-like potentials with higher field powers yield larger values for $n_{s}$, within the observationally allowed window, and that even for the case in study the addition of dissipative effects during inflation (leading to a warm inflation regime) can sufficiently increase the spectral index (see, e.g., Ref. [55]). We leave some of the details of the dynamics of inflation and observational predictions for these potentials to the appendix, and here we focus on the problem of initial conditions, which is the main scope of this work.

We have studied the dynamics of inflation for the non-renormalizable plateau potential, in particularly solving numerically the coupled inflaton and Friedmann equations so as to determine the initial conditions in phase space leading to a sufficiently long period of inflation. From this study, detailed in Appendix B, we have obtained the following constraints on the initial field value and velocity, respectively,

$$
\begin{aligned}
\left|\phi_{i}\right| / M_{P} & \lesssim 3 \times 10^{-2}\left(v / M_{P}\right)^{2}, \\
\left|\phi_{i}^{\prime}\right| / M_{P} & \lesssim 8 \times 10^{-2}\left(v / M_{P}\right)^{2},
\end{aligned}
$$

where $\phi^{\prime} \equiv d \phi / d N_{e}$. Taking into account that for this model $r \simeq 4 \times 10^{-7}\left(v / M_{P}\right)^{4}$ for $v \lesssim M_{P}$, the average values obtained for the field and velocity at the temperature $T_{e q}$, where the inflaton potential dominates over the radiation energy density, and given in Eqs. (3.10) 
and (3.11), can be written, respectively, as

$$
\begin{aligned}
\phi_{c} & \simeq 2 \times 10^{-10} \frac{g_{*}^{1 / 8}}{\alpha}\left(\frac{v}{M_{P}}\right)^{5 / 2} M_{P}, \\
\phi_{c}^{\prime} & \simeq 9 \times 10^{-7} g_{*}^{-1 / 8}\left(\frac{v}{M_{P}}\right)^{3 / 2} M_{P} .
\end{aligned}
$$

This shows that, with the pre-inflationary thermalization period, the average field value is always within the required range of initial conditions for any $v<M_{P}$, while the field velocity is within the required range for $10^{-8} M_{P} \lesssim v<M_{P}$. There is, thus, a wide parametric range for which inflation starts naturally after the initial radiation-dominated period.

We also note that the amplitude of the primordial curvature power spectrum $\Delta_{\mathcal{R}}^{2} \simeq$ $\left(2 / 3 \pi^{2}\right)\left(V_{0} / M_{P}^{4}\right) r^{-1} \simeq 2.2 \times 10^{-9}$ yields $\lambda=V_{0} / v^{4} \simeq 10^{-14}$, which is the typical value for inflaton self-interactions found generically in single-field inflation models. We will use this below to estimate the effects of nonlinearities in the field potential on the dynamics of the long-wavelength modes.

One aspect that we may immediately check is whether our assumption that the fermions coupled to the inflaton remain light throughout the initial radiation-dominated era is satisfied. If one assumes equipartition of energy at the end of the Planck era, as discussed earlier, we have an initial temperature $T \sim g_{*}^{-1 / 4} M_{P}$ parametrically below the Planck scale, and $V(\phi) \sim T^{4} \sim M_{P}^{4} / g_{*}$. In the presence of a thermal bath of relativistic particles, the leading thermal correction to the inflaton potential is the thermal mass term $\alpha^{2} T^{2} \phi^{2}$, as discussed earlier, and one can easily check that for $\lambda \sim 10^{-14}$ this term dominates over the zerotemperature part of the potential at such a high temperature for field values such that $V(\phi) \sim M_{P}^{4} / g_{*}$. This means that the typical value for the inflaton field at the end of the Planck era is $\phi \sim \alpha^{-1} T \ll g^{-1} T$ for small couplings and a sufficiently large number of species such that $\alpha \lesssim 1$. Hence, assuming an initial equipartition of energy, the fermion mass is initially below the temperature, and as we have shown earlier, it will then remain so throughout the radiation-dominated period.

We will start by assuming that equipartition holds, such that our thermalization and localization mechanism begins immediately after the Planck era. At the end of this section, we will nevertheless consider the alternative possibility that the inflaton carries a larger fraction of the energy balance as the Universe emerges from this putative quantum gravity regime, in which case the fermions are initially heavy and cannot be part of the relativistic thermal bath.

\subsection{Evolution after inflaton-radiation equality, thermal inflation and nonlinear effects}

We have shown that, in the radiation-dominated era prior to the onset of inflation, field modes can get localized close to the origin (plateau) through the combined effects of the induced thermal mass and fluctuation-dissipation terms in the equation of motion. We have concluded that the classical field component has a very small mean variance, $\left\langle\phi_{c}^{2}\right\rangle \simeq\left(6 \pi^{2} \alpha^{2}\right)^{-1} H^{3} / T \ll$ $T$, while the full field variance including sub-horizon momentum modes with $p \lesssim T$ is larger, $\left\langle\phi^{2}\right\rangle \sim\left(2 \pi^{2} \alpha^{2}\right)^{-1} T^{2} \sim T^{2}$. The energy in the sub-horizon modes redshifts as radiation, so it will not come to dominate over the constant plateau $V_{0}$ as long as the super-horizon classical field modes can be kept on top of the plateau for sufficiently long. Nevertheless, since there 
are nonlinear terms in the scalar potential, one must check whether the consequent mixing between sub- and super-horizon field modes can bring the latter outside the plateau and spoil the initial conditions for inflation.

First, let us note that the inflaton-radiation equality temperature $T_{e q}$ satisfies

$$
T_{e q}=\left(30 / \pi^{2}\right)^{1 / 4} g_{*}^{-1 / 4} V_{0}^{1 / 4} \ll v,
$$

since $V_{0}=\lambda v^{4}$ with $\lambda \ll 1$. This means the full inflaton field should be everywhere within the plateau region at equality, also giving $g \phi \ll T$, such that fermions are light as assumed.

The full effective potential at finite temperature can be written as:

$$
V_{e f f}=V_{0}\left[1-3(\phi / v)^{4}+2(\phi / v)^{6}\right]-\frac{2 N_{F}}{\pi^{2}} T^{4} \int_{0}^{\infty} d y y^{2} \log \left[1+\exp \left(-\sqrt{y^{2}+g^{2} \phi^{2} / T^{2}}\right)\right] .
$$

For relativistic fermions, $g|\phi| \ll T$, when including the leading thermal corrections, we obtain that

$$
V_{e f f} \simeq V_{0}\left[1-3(\phi / v)^{4}+2(\phi / v)^{6}\right]-\frac{7 \pi^{2}}{180} N_{F} T^{4}+\frac{1}{2} \alpha^{2} T^{2} \phi^{2},
$$

while thermal corrections are Boltzmann-suppressed for $g|\phi| \gtrsim T$. Note that the first term in this high temperature expansion is field independent and has a negative sign. This does not, however, correspond to a negative energy density, since the (free) energy density is $\rho=V_{\text {eff }}+T s$, where the entropy density is $s=-d V_{\text {eff }} / d T$. This yields a total field independent contribution of $\left(7 \pi^{2} / 60\right) N_{F} T^{4}$, which is the well-known contribution of $N_{F}$ relativistic Dirac fermions to the energy density of the thermal radiation bath, usually written as $\rho_{R}=\left(\pi^{2} / 30\right) g_{*} T^{4}$ with $g_{*}=(7 / 8) \times 4 N_{F}$.

Let us then study the change in the shape of the potential close to the equality temperature $T_{e q}$, which we illustrate in Fig. 2 (note that when plotting the effective potential, since $\phi$ covers values ranging from high temperature $g|\phi| \ll T$ to low temperatures $g|\phi| \gtrsim T$, it is not appropriate to use the result Eq. (4.8), but the full expression Eq. (4.7) or an approximation of it that covers both the high and low temperature regimes [56]).

We can see in this figure that, on the one hand, at large field values the effective potential coincides with the zero-temperature form, which is due to the fact that $g|\phi| \gtrsim T$ at large field values and thermal effects are exponentially suppressed. In a vicinity of the origin, on the other hand, there is a dip in the potential as a result of thermal effects, essentially corresponding to the thermal mass correction that we have considered in the pre-radiation era. This dip naturally becomes shallower and narrower as the temperature decreases, but it never actually disappears if the fermion mass vanishes at the origin, since there is always an arbitrary vicinity of the origin where the latter are relativistic and yield a thermal correction to the otherwise vanishing inflaton mass.

Despite the thermal dip in the potential becoming increasingly narrow, we have seen that fluctuation-dissipation effects yield an average thermal field fluctuations $\langle|\phi|\rangle \sim T$, for which $g|\phi|<T$ for small couplings. This means that the inflaton is localized in this shallow dip at the equality temperature, as shown in the zoomed inset plot in Fig. 2. At such average field values we indeed find for the first and second derivatives of $V_{\text {eff }}$ with respect to the field, respectively,

$$
\begin{aligned}
& V_{e f f}^{\prime}(\phi \sim T, T) \simeq\left(-12 \lambda+\alpha^{2}\right) T^{3}>0, \\
& V_{e f f}^{\prime \prime}(\phi \sim T, T) \simeq\left(-36 \lambda+\alpha^{2}\right) T^{2}>0,
\end{aligned}
$$




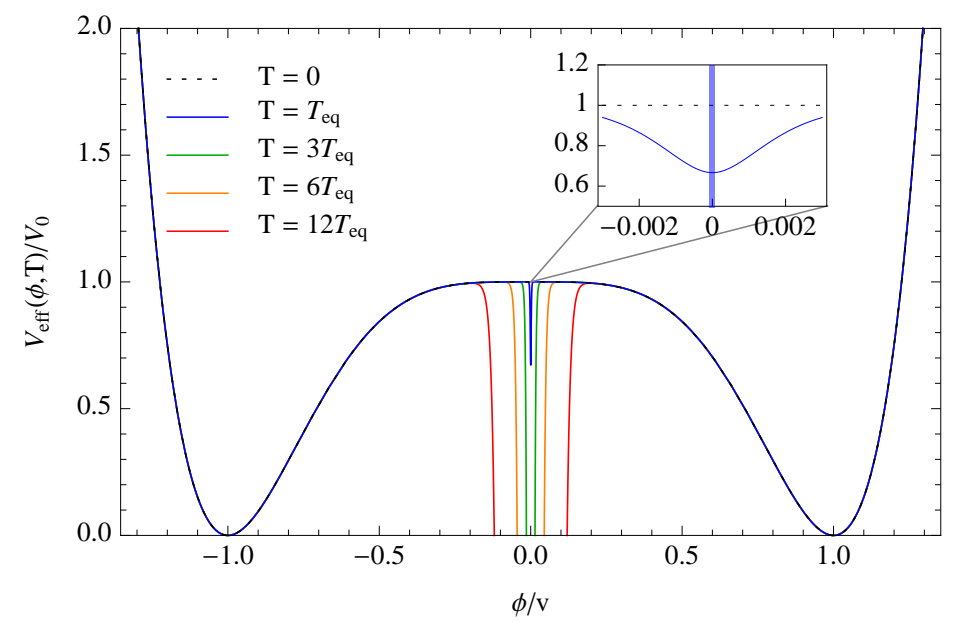

Figure 2. The effective plateau-like potential given by Eq. (4.7) for different temperatures above $T_{e q}$. The inset plot zooms in at the region about the origin for $T=T_{e q}$, with the narrow shaded blue region corresponding to $-T_{e q}<\phi<T_{e q}$ and hence to the average fluctuation range of the inflaton field at this temperature.

where we have used that $\alpha^{2} \gg \lambda$. This implies that the potential is dominated by the thermal mass for field values below the temperature. Hence, at $T_{e q}$ all field modes will still oscillate about the origin.

Since $V_{0}$ becomes dominant and the Hubble parameter becomes constant, $H_{\text {inf }} \simeq$ $\sqrt{V_{0} / 3 M_{P}^{2}}$, the condition $\Gamma_{\phi}>H_{\text {inf }}$ eventually fails and the field can no longer remain in thermal equilibrium, such that we expect the fluctuation-dissipation effects from the decay into fermions to become sub-dominant. The decoupling temperature at which $\Gamma_{\phi}=H_{\text {inf }}$ is then given by

$$
T_{D}=\frac{16 \pi}{3} \frac{\lambda}{\alpha^{4}} \frac{v^{2}}{M_{P}} \simeq 0.2\left(\frac{\alpha}{0.4}\right)^{-4}\left(\frac{\lambda}{10^{-14}}\right)^{1 / 4} g_{*}^{1 / 4}\left(\frac{v}{M_{P}}\right) T_{e q},
$$

which shows that thermalization may still proceed for temperatures parametrically below equality for $v \ll M_{P}$. Note that this does not imply that all dissipative effects shut down, since adiabatic dissipation (see, e.g., Ref. [45]) may still be significant and in fact lead to a period of warm inflation $[47,57]$, as we discuss in Appendix B, but let us for simplicity focus on the case where dissipative effects can be neglected below $T_{e q}$. As the thermal mass dominates the potential at $T_{e q}$ in the localized field range, all relevant momentum modes satisfy a linear equation of motion, which is given by

$$
\ddot{\phi}_{k}+3 H_{\text {inf }} \dot{\phi}_{k}+\omega_{k}^{2} \phi_{k}=0,
$$

where $\omega_{k} \simeq \alpha T$ for all momentum modes with $k \lesssim T$. As long as $T \gtrsim H_{\text {inf }}$, the modes remain underdamped and oscillating about the origin, and it is easy to check that the amplitude of each mode decays as $e^{-3 H t / 2}$, i.e., the whole field amplitude decays as $a^{-3 / 2}$. Since $T \propto 1 / a$, the field amplitude decreases faster than the temperature, and one remains in the regime where $g|\phi|<T$ and where the thermal mass dominates the potential until the temperature drops below $H_{\text {inf }}$. This is, in fact, essentially a period of thermal inflation [58, 59] following the pre-inflationary radiation-dominated era. One can also see that this could 
have an arbitrarily long duration, since even though the temperature is decreasing, the field gets more and more localized close to the origin, where there is a local minimum (note that this is a feature of plateau-like potentials where the zero-temperature field mass vanishes at the origin). This period may, however, end before the temperature falls below the Hubble parameter, if the fermions have a field-independent mass $m_{f}>H_{\text {inf }}$, such that thermal effects become exponentially suppressed for any field value for $T \lesssim m_{f}$. We conclude that the average inflaton field value at $T \sim m_{f}$ should then be given by

$$
\left\langle\phi^{2}\right\rangle \simeq T_{e q}^{2}\left(\frac{m_{f}}{T_{e q}}\right)^{3}
$$

where we used that $\phi \propto a^{-3 / 2} \propto T^{3 / 2}$. At this stage, the nonlinear zero-temperature terms in the inflaton potential become dominant and the inflaton field will classically roll towards the true minimum at $\phi= \pm v$. To ensure that the classical component can follow a slow-roll trajectory towards the minimum, we may evaluate the slow-roll parameters at this average field value, yielding in particular, that

$$
\eta_{\phi} \simeq-36\left(\frac{M_{P}}{v}\right)^{2}\left(\frac{T_{e q}}{v}\right)^{2}\left(\frac{m_{f}}{T_{e q}}\right)^{3}
$$

where $\eta_{\phi}=M_{P}^{2} V^{\prime \prime} / V$ as usual. If one takes, e.g., $m_{f} \sim H_{\text {inf }}$, this yields

$$
\left|\eta_{\phi}\right| \sim 10^{-17} g_{*}^{1 / 4}\left(\frac{v}{M_{P}}\right) \ll 1,
$$

when taking $\lambda \sim 10^{-14}$. This implies that nonlinear effects cannot spoil inflation along the plateau-like potential for any value of $v<M_{P}$. The field is then extremely localized at the origin after this period of thermal inflation, such that a long period of slow-roll inflation should follow.

The duration of the period of thermal inflation is model dependent, being set by the ratio $T_{e q} / m_{f}$ according to the above discussion. We do not expect this period to have any direct observational impact, since curvature perturbations exiting the horizon during this period have a temperature dependent spectrum that cannot be nearly scale-invariant due to the exponentially fast decrease in the temperature. Hence, the relevant CMB perturbations must be generated during the subsequent slow-roll phase, which must last for 50-60 e-folds independently of the duration of the previous thermal inflation phase. Nevertheless, thermal inflation sets the temperature at which slow-roll begins and thus the initial state for inflaton fluctuations, which differs from the Bunch-Davies vacuum. This may then lead to distinctive observational features that we plan to analyze in detail in future work.

We note that the analysis above is purely classical, neglecting in particular the possibility of quantum tunneling. Although this possibility is hard to analyze in detail, we note that for tunneling into the true minimum of the potential to occur, one requires a field fluctuation that places the inflaton outside the range where the thermal mass is dominant. For $T \gtrsim m_{f}$, this implies a field fluctuation such that the fermions become heavy, $\delta \phi \sim T / g$. For $g \ll 1$, such a fluctuation is much larger than the average thermal field fluctuations, $\sqrt{\left\langle\phi^{2}\right\rangle} \sim T$, so we expect the probability of quantum tunneling to be suppressed for small couplings and parametrically large field multiplicities such that $\alpha \sim g \sqrt{N_{F}} \lesssim 1$. 


\subsection{An initial period of chaotic inflation for large field amplitudes followed by inflation on the plateau}

We note from the results of the previous subsection that the thermal mass is the leading correction to the potential for $g|\phi|<T$. Other corrections in the high-temperature expansion of the effective potential (see, e.g., Ref. [60]) include, for example, Coleman-Weinberg terms at finite temperature that induce quartic self-interactions of the inflaton field with an effective coupling $\lambda_{e f f} \sim g^{4} N_{F}$ up to loop suppression factors. These corrections to the quartic coupling, generated by the loop thermal corrections to the tree-level potential, may exceed the zero-temperature coupling $\lambda \sim 10^{-14}$ (noting that at zero-temperature Coleman-Weinberg corrections may be suppressed by supersymmetry or decoupling effects as discussed previously, in Subsec. 3.1). These induce nonlinear effects that mix the different field modes, but are generically suppressed compared to the effect of the thermal mass and, hence, they may be discarded. For instance, we have that

$$
\frac{\lambda_{e f f} \phi^{4}}{\alpha^{2} T^{2} \phi^{2}} \sim \frac{g^{4} N_{F} \frac{T^{4}}{\alpha^{4}}}{T^{4}} \sim N_{F}^{-1} \ll 1
$$

Although this shows that generically our analysis should be valid already at the Planck era, we cannot exclude the possibility that the potential is dominated by the zero-temperature power-law term ( $\phi^{6}$ in the present case-study) at the Planck era. This may occur in particular if, for some reason, equipartition of energy at the Planck era does not apply and the inflaton energy density exceeds that of the thermal bath. In this case we may have $g|\phi| \gtrsim T$ at the Planck era, such that the potential has the zero-temperature form.

This possibility is quite interesting, since in this case the field value will typically be sufficiently large to trigger a period of chaotic inflation with a power-law potential already at the Planck era. Note that $H \sim M_{P}$ at the Planck era, implying that only super-planckian modes are within the horizon at this stage. Assuming that the typical physical momentum of the excited field modes is at most $k \lesssim M_{P}$, we conclude that typical modes are superhorizon at this stage and so chaotic inflation will be triggered if the slow-roll coefficient $\epsilon_{\phi}=M_{P}^{2}\left(V^{\prime} / V\right)^{2} / 2<1$ (note that chaotic inflation is an attractor in phase space when this condition is satisfied). For a $\phi^{6}$ potential this condition corresponds to $|\phi|>3 \sqrt{2} M_{P} \sim g_{*}^{1 / 4} T$ at the Planck era, which is easily satisfied if $|\phi| \gtrsim T / g$ and thermal effects are sub-dominant.

If chaotic inflation begins at the Planck era, we may expect the thermal bath to be diluted away very quickly. Nevertheless, this inflationary period will last until $\epsilon_{\phi} \sim 1$. At the end of this initial slow-roll regime, it is easy to see that $V \sim V_{0}\left(M_{P} / v\right)^{6} \gg V_{0}$. This large energy density will then be transferred into radiation through the standard reheating process as the field oscillates about the minimum(a) at $\phi= \pm v$ and decays into fermions. If reheating is sufficiently fast (as will be the case if the parametric resonance instability of preheating [61] is effective), the radiation energy density will largely exceed $V_{0}$ after this reheating period, and the plateau potential may once more be lifted by thermal effects.

The reheating process may be quite involved, including both perturbative and nonperturbative effects (see, e.g., Refs. [62, 63] for recent reviews), initially at nearly vanishing temperature but then at finite temperature once the fermions that result from the inflaton decay thermalize. Nevertheless, we may have a good idea of the parametric regimes required to successfully reheat the Universe and thermalize the inflaton by considering first the $T=0$ perturbative decay of the inflaton into fermions.

Since the model Eq. (4.1) is dominated by the non-renormalizable $\phi^{6}$ term after inflation, we can assume this form for the potential in the initial phase of oscillations. Let us also assume 
that reheating will occur while the inflaton amplitude is still sufficiently large that we may discard other terms. In this case, the inflaton mass is $m_{\phi}=\sqrt{V^{\prime \prime}(\phi)}=\sqrt{60 \lambda} \phi^{2} / v$, while the fermion mass is $m_{f}=g|\phi|$. Inflaton decay is then kinematically possible once $m_{\phi}>2 m_{f}$, which happens for field amplitudes

$$
|\phi|>\frac{1}{\sqrt{15}} \frac{g}{\sqrt{\lambda}} v
$$

which is parametrically larger than the minimum $v$, since $\lambda \sim 10^{-14}$ for observationally consistent models, unless the coupling $g$ is quite suppressed. For field amplitudes larger than this value, we may neglect the fermion masses, such that the zero-temperature perturbative decay width is given by

$$
\Gamma_{\phi}=\frac{g^{2} N_{F}}{8 \pi} m_{\phi}=\frac{3 \alpha^{2}}{2 \pi}(\sqrt{15 \lambda}) \frac{\phi^{2}}{v} .
$$

For a field oscillating in a $\phi^{n}$ potential, the average kinetic and potential energies are related by the virial theorem, $\langle K\rangle=(n / 2)\langle V\rangle$, such that in our case the average energy density of the oscillating inflaton is

$$
\left\langle\rho_{\phi}\right\rangle=4\langle V(\phi)\rangle=8 \lambda \frac{\phi^{6}}{v^{2}} .
$$

Since this is the dominant component before the inflaton decays significantly, this yields an average Hubble parameter given by

$$
H=\sqrt{\frac{8}{3}} \sqrt{\lambda} \frac{\left|\phi^{3}\right|}{v M_{P}} .
$$

Decay will then occur when $\Gamma_{\phi}>H$, i.e., for field amplitudes satisfying

$$
|\phi|<\phi_{R} \equiv \frac{9}{4 \pi} \sqrt{\frac{5}{2}} \alpha^{2} M_{P} \sim \alpha^{2} M_{P} .
$$

Since we require $\alpha \gtrsim 0.1$ for thermalization of the inflaton after reheating, this is just below $M_{P}$ and for $v \ll M_{P}$ we may safely discard other terms in the potential as we assumed above. We must, for consistency, require that the decay is kinematically possible at reheating, at $|\phi|=\phi_{R}$, according to the constraint Eq. (4.17), yielding the upper bound:

$$
\frac{v}{M_{P}}<\frac{45}{4 \pi} \sqrt{\frac{3}{2}} \alpha^{2}\left(\frac{\sqrt{\lambda}}{g}\right)
$$

which may also be regarded as an upper bound on the Yukawa coupling $g$. Note that if this is satisfied, decay will be kinematically possible at the end of inflation with $|\phi| \sim \sqrt{18} M_{P} \gg \phi_{R}$.

When $\Gamma_{\phi} \sim H$, the inflaton will effectively decay and the Universe becomes dominated by the inflaton's decay products. Assuming that these decay products are light (as we will explicitly check below) and that they thermalize quickly through processes other than the interactions with the inflaton field (e.g., through their interactions with gauge particles), we can estimate the reheating temperature $T_{R}$ by equating $\Gamma_{\phi}$ with the Hubble parameter in a radiation-dominated Universe, which yields for $T_{R}$ the result

$$
T_{R}=\frac{135}{4 \pi^{2}}\left(\frac{3}{8}\right)^{1 / 4} \alpha^{3} g_{*}^{-1 / 4} \lambda^{1 / 4}\left(\frac{M_{P}}{v}\right)^{1 / 2} M_{P} \simeq 2 \alpha^{3}\left(\frac{M_{P}}{v}\right)^{3 / 2} T_{e q},
$$


which is thus parametrically larger than the equality temperature, $T_{e q}$, at which $V_{0}$ dominates over the radiation energy density for $\alpha \lesssim 1$ and $v \ll M_{P}$. The upper bound on $v / M_{P}$ in fact yields a lower bound on the reheating temperature, given by $T_{R} \gtrsim 7\left(g / 10^{-6}\right)^{3 / 2} T_{e q}{ }^{4}$

Now, we must check that the fermions are indeed light at the field amplitude and temperature at reheating. From the above results for $\phi_{R}$ and $T_{R}$, we readily find that

$$
\frac{g \phi_{R}}{T_{R}}=\frac{\pi}{3 \sqrt{5}}\left(\frac{2}{3}\right)^{1 / 4} \alpha^{-1} g_{*}^{1 / 4}\left(\frac{g}{\lambda^{1 / 4}}\right)\left(\frac{v}{M_{P}}\right)^{1 / 2} \lesssim 2 \sqrt{\alpha}
$$

where in the last step we have used the upper bound on $v / M_{P}$ obtained in Eq. (4.22). We thus see that the fermion mass at reheating is at most of the order of the temperature, so that the calculation is consistent. This also means that the thermalized fermions will then uplift the potential with the thermal mass correction, with the condition $g|\phi| / T$ satisfied at all subsequent times, and localize the field at the origin, such that a second period of inflation along the plateau may occur when the temperature eventually falls below $T_{e q}$.

We note that the consistency of this analysis requires essentially that the upper bound on $v / M_{P}$, Eq. (4.22), is satisfied, which for $\lambda \sim 10^{-14}$, can be written as

$$
\frac{v}{M_{P}} \lesssim 10^{-7} \alpha \sqrt{N_{F}}
$$

We note that this analysis neglects thermal corrections to the inflaton decay width, which will be active as soon as a significant density of thermalized fermions is achieved before reheating is complete, and also an initial stage of preheating, which may change these constraints. Nevertheless, we see that it is parametrically possible to reheat the Universe after the period of chaotic inflation so as to restore the symmetry and induce a subsequent period of inflation along the plateau part of the potential.

The possibility of having two periods of inflation is quite attractive, since if chaotic inflation is triggered immediately after the Planck era any initial curvature in the Universe will be diluted away before the second period of inflation along the plateau. In the absence of such a period of chaotic inflation, there may be a too long period of radiation-domination before $V_{0}$ becomes dominant, and if the Universe has initially a positive curvature it may overclose before inflation begins. This implies that some degree of tuning of the initial curvature of the Universe is required for a successful inflationary model with a plateau-like potential, which is naturally less attractive.

\section{Conclusions}

In this work we have analyzed the possibility of addressing the initial conditions problem for inflation with a plateau-like potential with finite temperature fluctuation-dissipation effects. We have shown that in a pre-inflationary radiation-dominated era the coupling between the inflaton and a thermal bath of relativistic fermions induces (i) a thermal mass correction that dominates the potential, (ii) a friction term corresponding to the inflaton decay into

\footnotetext{
${ }^{4}$ We note that non-perturbative effects, in particular a parametric resonance, may speed up the reheating process and should be taken into account in a more accurate treatment, which we leave for future work. However, backreaction effects necessarily shut down the parametric resonance once the produced particles have an energy density comparable to the inflaton's energy density, and reheating always ends in the perturbative regime. Considering only the perturbative decay of the inflaton thus yields an accurate estimate of the reheating temperature.
} 
fermions and (iii) an associated noise term that sources inflaton fluctuations. The combined result of these effects is a localization of inflaton modes about the origin, particularly the super-Hubble modes, while sub-Hubble modes acquire a thermal spectrum.

This localization effect is a direct consequence of fluctuation-dissipation dynamics driving inflaton modes towards a thermal equilibrium distribution, reproducing the results found in [14] where such a distribution was assumed. One should note that this is a non-trivial result since, while local thermal equilibrium is a valid assumption on short sub-horizon scales, nothing can a priori be said about the inflaton mode distribution on large scales. Employing the framework of fluctuation-dissipation dynamics, we have shown that super-horizon inflaton modes evolve towards an equilibrium distribution as a consequence of thermal and dissipative effects on short scales.

The formalism of fluctuation dissipation has also allowed us to conclude that a localization of the inflaton's long wavelength modes occurs provided that the coupling between the inflaton and the species in the radiation bath is sufficiently large for thermalization to occur before the plateau potential comes to dominate the energy balance. The lower bound on the coupling decreases with the height of the potential, $V_{0}$, since inflaton-radiation equality occurs later for smaller $V_{0}$, but we have found that the effective coupling $\alpha=g \sqrt{N_{F} / 6}>\mathcal{O}(0.1)$ unless the tensor-to-scalar ratio is very suppressed. Such effective couplings could, of course, be the result of a large number of species in the thermal bath and not necessarily of large individual couplings.

While it is commonly believed that such large couplings can spoil the flatness of the potential and its observational predictions, we have argued that in supersymmetric models, for example, effective couplings of the required size do not modify the tree-level potential in a significant way, thus consistently allowing for the localization of the inflaton at the origin through thermal fluctuation-dissipation effects before the onset of the slow-roll regime, independently of whether the latter occurs in a cold or warm regime. The addition of scalar degrees of freedom will, in fact, help the thermalization process. We have also pointed out that, even in the absence of supersymmetry, sufficiently large couplings may be allowed provided that the species responsible for the inflaton thermalization become heavy during the slow-roll regime, compared to the relevant energy scale during inflation, which should arguably be the inflationary Hubble scale. The quantum corrections to the scalar potential induced by these heavy species are then suppressed compared to the conventional ColemanWeinberg term, in agreement with the decoupling theorem. We note that this is naturally seen using a mass-dependent renormalization scheme, while decoupling has to be enforced as a separate constraint in mass-independent schemes, such as the conventionally used minimal subtraction procedure.

We have also shown that equipartition of energy at the end of the Planck era generically leads to initial field values such that the fermionic species in the thermal bath are relativistic and that the field evolution is such that they remain relativistic throughout the radiation-dominated era and even after inflation begins. In fact, for temperatures above the bare (i.e. field-independent) fermion mass, $T \gtrsim m_{f}$, thermal corrections dominate the inflaton potential close to the origin and prevent slow-roll from starting. If $m_{f}<T_{e q}$, we have thus found that the pre-inflationary radiation era is followed by a period of thermal inflation. This implies that the slow-roll regime, where the final 50-60 e-folds of inflation should occur, starts at a much lower temperature than previously thought. This in turn suppresses the non-linear mixing between super-Hubble and thermalized sub-Hubble modes that could otherwise prevent the onset of slow-roll. We have also shown that the energy stored in the 
sub-Hubble modes redshifts like radiation, so that they are effectively a part of the thermal radiation bath and become sub-dominant at $T_{e q}$. We note that dissipative effects can still be significant during this first thermal inflation stage, until the inflaton decay rate drops below the expansion rate, and in this sense there is also an early warm inflation period even if fluctuation-dissipation effects play no role in the subsequent slow-roll dynamics.

If equipartition of energy does not hold at the Planck scale and the inflaton carries a larger fraction of the energy density, one is likely to start in a regime where the fermions are heavy and do not induce thermal effects on the scalar potential. Unless the plateau section of the potential extends to such large (and most probably super-planckian) field values, the field will naturally be outside the plateau region where the potential has a monomial form, as in the examples considered in this work. It is then likely that a first period of chaotic inflation may be triggered at the Planck era. At the end of this period, the energy density in the inflaton still largely exceeds the height of the sub-Planckian plateau, so that after reheating one may enter a radiation-dominated era during which the inflaton gets localized at the origin by fluctuation-dissipation effects and a second period of slow-roll inflation along the plateau may follow. CMB observations will only be sensitive to the final period, but this is an attractive scenario since it allows for inflation starting immediately after the Planck era while yielding a sufficiently low tensor-to-scalar ratio.

Hence, the thermalization and localization mechanism described in this work can be relevant whether there is already a thermalized bath at the end of the Planck era or if inflation is triggered from chaotic initial conditions at this stage, producing a thermal bath upon a first reheating stage. The first possibility should occur if the initial inflaton field value is sufficiently small for the fermions it interacts with to be light at this stage, provided of course that thermalization of these degrees of freedom can occur in a Planck regime, while chaotic inflation should be triggered in patches of the Universe where the inflaton field value is larger and the fermions are most likely too heavy to form a thermalized bath. Both possibilities are available in potentials with a flat section near the origin and a monomial behavior at large field values.

While several of our conclusions were derived in the context of a particular plateaulike potential, we expect the thermalization and localization mechanism to apply to any potentials with a plateau-like region about the origin such that $V^{\prime}(0)=V^{\prime \prime}(0)=0$, and which require fine-tuned initial conditions to trigger a sufficiently long period of slow-roll inflation. Thermal fluctuation-dissipation effects yield a natural dynamical mechanism to obtain such initial conditions and, as we have shown in this work, can be significant without leading to large quantum corrections to the inflaton potential, as commonly believed. While there are potentially other aspects of the initial condition problem at the non-linear level that we cannot address in this framework, our conclusions show that the inflationary paradigm is not necessarily in trouble with the latest constraints on the tensor-to-scalar ratio, even without considering modifications of the gravity theory. Furthermore if the inflationary period is just long enough to solve the horizon problem, it has been recognized $[64,65]$ that remnants of the pre-inflationary era might leave imprints on the data. The pre-inflation fluctuation-dissipation dynamics studied in this paper could be of interest to examine in this context. We thus hope that this work motivates further consideration of plateau-like models in particle physics scenarios where the generic mechanism outlined here can be implemented. 


\section{Acknowledgments}

M.B.G. is partially supported by "Junta de Andalucía" (FQM101) and the University of Granada (PP2015-03). A.B. is supported by STFC. R.B. wishes to thank the Institute for Theoretical Studies of the ETH Zürich for kind hospitality. R.B. acknowledges financial support from Dr. Max Rössler, the Walter Haefner Foundation, the ETH Zürich Foundation, and from a Simons Foundation fellowship. The research of R.B. is also supported in part by funds from NSERC and the Canada Research Chair program. I.G.M. is partially supported by the UK Science and Technology Facilities Council Consolidated Grant ST/J000426/1. R.O.R is partially supported by research grants from Conselho Nacional de Desenvolvimento Científico e Tecnológico (CNPq), grant No. 303377/2013-5, Fundação Carlos Chagas Filho de Amparo à Pesquisa do Estado do Rio de Janeiro (FAPERJ), grant No. E - 26 / 201.424/2014, and Coordenação de Pessoal de Nível Superior - CAPES (Processo No. 88881.119017/201601). J.G.R. is supported by the FCT Investigator Grant No. IF/01597/2015 and partially by the H2020-MSCA-RISE-2015 Grant No. StronGrHEP-690904 and by the CIDMA Project No. UID/MAT/04106/2013. The authors also acknowledge the kind hospitality and support of the Higgs Centre for Theoretical Physics at the University of Edinburgh.

\section{A Inflation with the Higgs-like symmetry breaking potential}

In this appendix we analyze the inflationary dynamics for a Higgs-like potential of the form

$$
V(\phi)=\frac{\lambda}{4}\left(\phi^{2}-v^{2}\right)^{2}
$$

For this potential, the slow-roll parameters are given by

$$
\epsilon_{\phi}=8\left(\frac{M_{P}}{v}\right)^{2} \frac{x^{2}}{\left(1-x^{2}\right)^{2}}, \quad \eta_{\phi}=-4\left(\frac{M_{P}}{v}\right)^{2} \frac{1-3 x^{2}}{\left(1-x^{2}\right)^{2}}
$$

where $x=\phi / v$. From this we immediately see that the potential suffers from an "etaproblem" and cannot sustain a long period of inflation for $v<2 M_{P}$ even if the field is initially very close to the origin. We thus expect a successful period of inflation to occur only for super-planckian values of $v$. This is similar to chaotic inflation models, where the slowroll conditions are only satisfied for super-planckian field values, but in this case inflation may actually occur for sub-planckian field values, the condition $v \gtrsim M_{P}$ being essentially a condition on the curvature of the potential near the origin.

The number of e-folds of inflation after the present CMB scales become super-horizon at a field value $\phi_{*}$ is given by

$$
N_{e} \simeq-\int d \phi \frac{V(\phi)}{V_{, \phi}(\phi)}=\frac{v^{2}}{8 M_{P}^{2}}\left[\ln \left(\frac{x_{e}^{2}}{x_{*}^{2}}\right)+x_{*}^{2}-x_{e}^{2}\right],
$$

where the final field value $\phi_{e}$ corresponds to the smallest field value for which $\epsilon_{\phi}=1$. In Fig. 3 we plot the field value at horizon-crossing for 50-60 e-folds of inflation. As one can easily see, the last 50-60 e-folds of inflation will occur with super-planckian fields for $v \gtrsim 10 M_{P}$.

The spectral index and tensor-to-scalar-ratio are given, respectively, by

$$
\begin{aligned}
n_{s} & =1-6 \epsilon_{\phi_{*}}+2 \eta_{\phi_{*}}=1-8\left(\frac{M_{P}}{v}\right)^{2} \frac{4 x_{*}^{2}+1}{\left(x_{*}^{2}-1\right)^{2}}, \\
r & =16 \epsilon_{\phi_{*}}=48\left(\frac{M_{P}}{v}\right)^{2} \frac{x_{*}^{2}}{\left(x_{*}^{2}-1\right)^{2}} .
\end{aligned}
$$




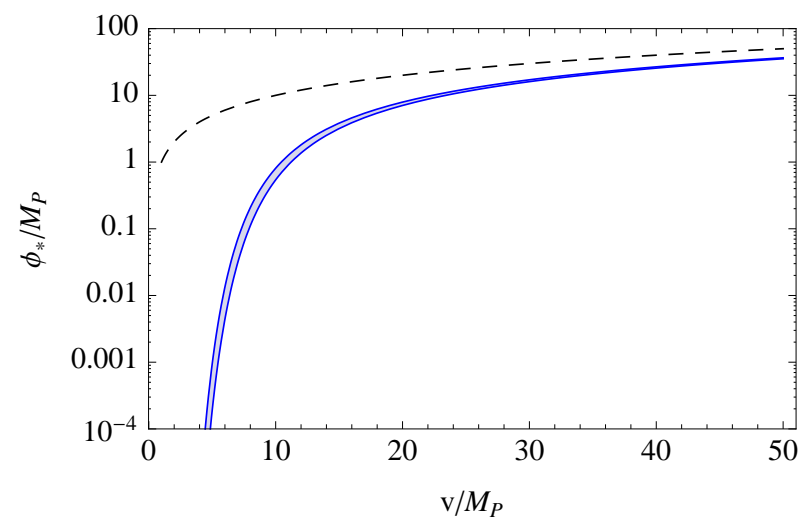

Figure 3. Field value at horizon-crossing yielding 50-60 e-folds of inflation as a function of $v$. For comparison, the dashed black line gives the value of $v$.

In Fig. 4 we show the observational predictions for $50-60$ e-folds of inflation after horizoncrossing and the constraints obtained by the Planck collaboration [4]. It is clear in Fig. 4 that the model is only in agreement with observations for $15 M_{P} \lesssim v \lesssim 40 M_{P}$, which corresponds to the regime where the observable e-folds of inflation occur for super-planckian field values.

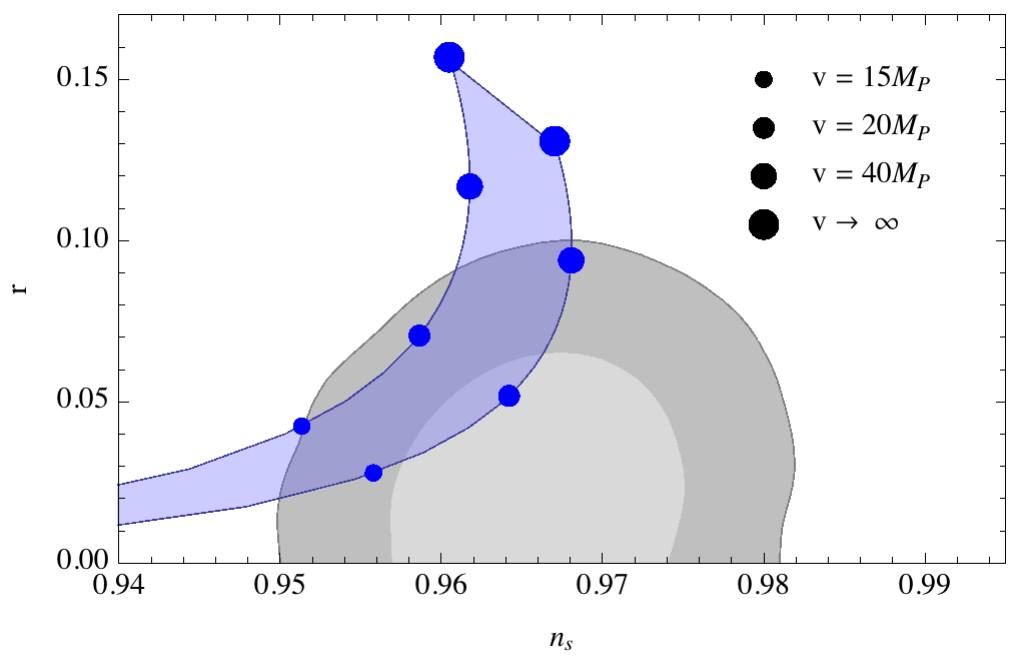

Figure 4. Trajectories in the $\left(n_{s}, r\right)$ plane for the symmetry-breaking potential with $50-60$ efolds of inflation (shaded blue region), along with the $68 \%$ and $95 \%$ C.L. obtained by the Planck collaboration. The circles give the prediction for particular values of $v$.

With the observational constraints on the value of the inflaton field VEV in mind, we may now discuss the initial conditions in phase space that lead to a sufficiently long period of inflation. We have solved the scalar field equation,

$$
\ddot{\phi}+3 H \dot{\phi}+V_{, \phi}(\phi)=0
$$

for different initial conditions in phase space and for different values of $v$. We show in Fig. 5 the regions in the plane $\left(\phi_{i}, \phi_{i}^{\prime}\right)$, with the subscript $i$ denoting the initial value and $\phi^{\prime}=\dot{\phi} / H$, where the slow-roll condition $\epsilon_{\phi}<1$ fails after more than 60 e-folds. 


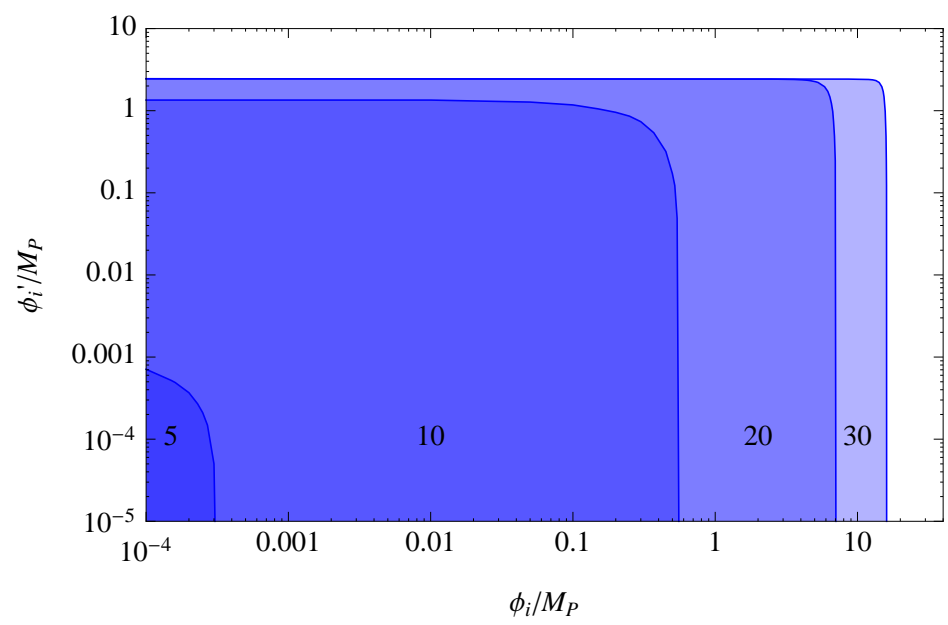

Figure 5. Regions in the initial phase space plane $\left(\phi_{i}, \phi_{i}^{\prime}\right)$ for which inflation lasts more than 60 e-folds for different values of $v / M_{P}$ indicated by the numbers inside each region.

As one can clearly see in Fig. 5, initial conditions leading to a sufficiently long period of inflation need to be significantly more tuned for smaller values of $v$, where the curvature of the potential near the origin is larger. This is the origin of the statement often found in the literature that "new inflation" potentials, as the symmetry-breaking potential example under consideration, require extremely fine-tuned initial conditions, but as one can clearly see this applies only to small values of $v$.

On the other hand we have seen above that observational constraints require superPlanckian values, in which case enough inflation may occur for a wide range of initial conditions. For large $v$, even super-planckian field values and velocities lead to a sufficiently long inflationary regime, with $\phi^{\prime}<\sqrt{6} M_{P}$ for $v \gg M_{P}$. In fact, in this case the inflationary trajectory is an attractor. We show this in Fig. 6 , where we plot several trajectories in the $(\phi, \dot{\phi})$ plane. We have used a rescaled time coordinate $\tilde{t}=\sqrt{\lambda} t$ which eliminates the dependence of the field equation on the self-coupling $\lambda$.

As one can see in Fig. 6, for $v=20 M_{P}$, panel (b), all trajectories with $\phi \lesssim v$ have a common straight portion corresponding to the slow-roll solution, showing that this is an attractor. This is opposed to the case $v=M_{P}$, panel (a), where no trajectory actually follows the slow-roll solution $\dot{\phi}=-V_{, \phi} / 3 H$. This is because for $v=M_{P}$ we have $\left|\eta_{\phi}\right|>1$, so that there are no consistent slow-roll solutions.

We thus conclude that the statement in the literature than "new inflation" requires much more fine-tuning of initial conditions than chaotic inflation is connected with the assumption that $v<M_{P}$. This thus seems a wrong comparison, since chaotic inflation requires superplanckian field values to allow for a wide range of initial conditions that yield a sufficiently long period of inflation. Whether the fact that the required field values are super-planckian poses a problem is still an object of debate, in the absence of a consistent ultra-violet completion. There are, on the one hand, those who argue (see, e.g., Ref. [66]) that, since the relevant energy density and curvature of the potential are sub-planckian, there is not any real problem in terms of putative quantum gravity corrections to the potential. On the other hand, there 


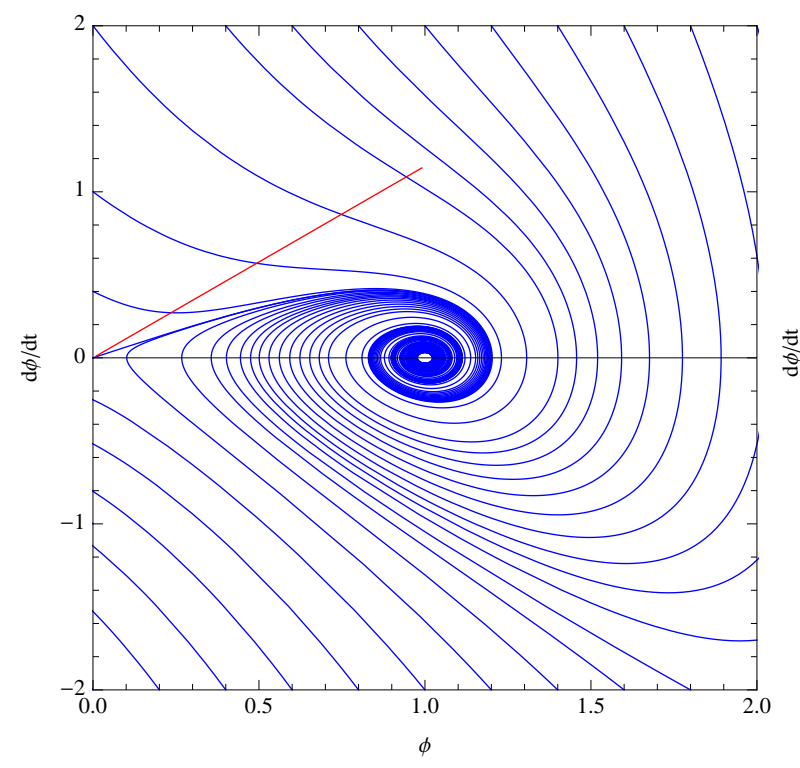

(a)

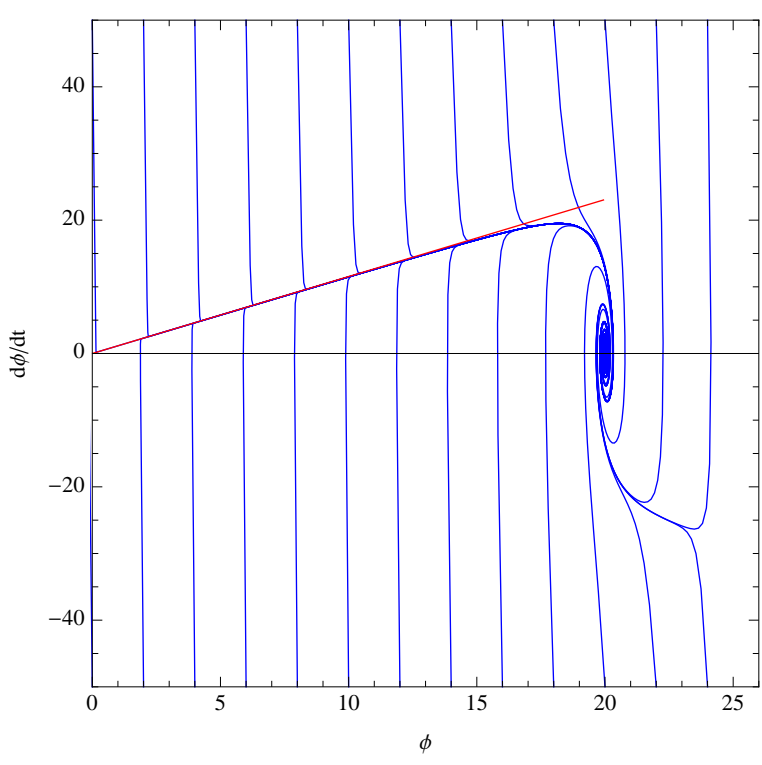

(b)

Figure 6. Phase space trajectories (shown in $M_{P}$ units) for $v=M_{P}$, panel (a) and for $v=20 M_{P}$, panel (b). Time is rescaled by a factor $\sqrt{\lambda}$ with respect to cosmic time. The red line gives, in each case, the slow-roll trajectory.

is a worry that such corrections may involve powers of $\phi / M_{P}$, in which case the dynamics of inflation in the super-planckian regime may not be under control. We do not aim to solve this problem, but we point out that the problem is as severe for new and chaotic inflation and that, provided that super-planckian values are allowed, both cases may yield a sufficiently long period of accelerated expansion for a wide range of initial conditions.

\section{B Non-renormalizable plateau-like potential}

The example of the symmetry breaking potential is, as we have seen above, only observationally consistent for $v>M_{P}$, for which the slow-roll solution is an attractor for a wide range of initial field and velocity values. In this sense, the initial condition problem is not very severe in this case. Let us now explore a different example, based on a non-renormalizable potential given by

$$
V(\phi)=V_{0}\left(1-\frac{m}{m-n} x^{n}+\frac{n}{m-n} x^{m}\right), \quad x=\phi / v,
$$

for $m>n$ and where $m>4$ and these potentials have minima at $\phi= \pm v$ with zero cosmological constant. Let us first investigate the case with $m=6$ and then the case of general (integer) power $m$.

\section{B.1 Plateau-like potential with sextic power}

Considering $m=6$ and $n=4$ in Eq. (B.1), we obtain

$$
V(\phi)=V_{0}\left(1-3 x^{4}+2 x^{6}\right), \quad x=\phi / v,
$$


and its shape is illustrated in Fig. 1. The slow-roll parameters for this potential are given by

$$
\epsilon_{\phi}=72\left(\frac{M_{P}}{v}\right)^{2}\left[\frac{x^{3}\left(x^{2}-1\right)}{1-3 x^{4}+2 x^{6}}\right]^{2}, \quad \eta_{\phi}=12\left(\frac{M_{P}}{v}\right)^{2}\left[\frac{x^{2}\left(5 x^{2}-3\right)}{1-3 x^{4}+2 x^{6}}\right]
$$

such that both parameters vanish at the origin, making it a flatter plateau than the Higgs-like potential. The number of e-folds of inflation after horizon-crossing is given by

$$
N_{e}=\frac{1}{12}\left(\frac{v}{M_{P}}\right)^{2}\left[\frac{1}{2 x_{*}^{2}}-\frac{1}{2 x_{e}^{2}}+x_{*}^{2}-x_{e}^{2}-\ln \left(\frac{x_{*}}{x_{e}}\right)\right],
$$

where $x_{e} \equiv \phi_{e} / v$ is the real solution of $\epsilon_{\phi}=1$ closer to the origin (we choose positive $x$ for concreteness using the reflection symmetry of the potential).

The spectral index and tensor-to-scalar ratio are given, respectively, by

$$
\begin{aligned}
n_{s} & =1-432\left(\frac{M_{P}}{v}\right)^{2}\left[\frac{x^{3}\left(x^{2}-1\right)}{1-3 x^{4}+2 x^{6}}\right]^{2}+24\left(\frac{M_{P}}{v}\right)^{2}\left[\frac{x^{2}\left(5 x^{2}-3\right)}{1-3 x^{4}+2 x^{6}}\right], \\
r & =1152\left(\frac{M_{P}}{v}\right)^{2}\left[\frac{x^{3}\left(x^{2}-1\right)}{1-3 x^{4}+2 x^{6}}\right]^{2} .
\end{aligned}
$$

It is then easy to see that for $v \ll M_{P}$ we have $x_{*} \ll 1$ and that $n_{s}-1 \simeq 3 / N_{e}$, which yields $n_{s}=0.94-0.95$ for $50-60$ e-folds of inflation and an extremely small tensor-to-scalar ratio $r \simeq\left(v / M_{P}\right)^{4} /\left(12 N_{e}^{3}\right) \ll 1$. Although the values of the spectral index in this subplanckian regime are just outside the $95 \%$ C.L. Planck contour, as shown in Fig. 7, allowing for a slightly larger number of e-folds would bring this potential into agreement with the data. As one can see, for $10 M_{P} \lesssim v \lesssim 20 M_{P}$ there is a good agreement with the data, but this case is similar to the Higgs-like potential analyzed above and we do not expect a severe initial condition problem in this regime. We will then focus on the $v<M_{P}$ regime.

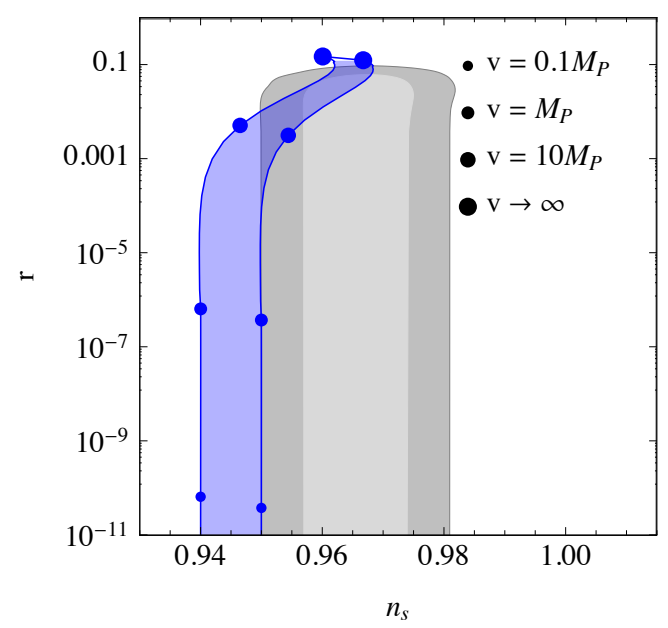

Figure 7. Trajectories in the $\left(n_{s}, r\right)$ plane for the non-renormalizable plateau-like potential with $50-60$ efolds of inflation (shaded blue region), along with the $68 \%$ and $95 \%$ C.L. obtained by the Planck collaboration. The circles give the prediction for particular values of $v$.

As for the Higgs-like potential, we have solved the (homogeneous) scalar field equation (A.6) numerically for different initial conditions in phase space, searching for values for which 
slow-roll lasts more than 60 e-folds. The results are illustrated in Fig. 8. It is clear that, for smaller $v$, one requires the field to be initially closer to the origin and with a smaller velocity.

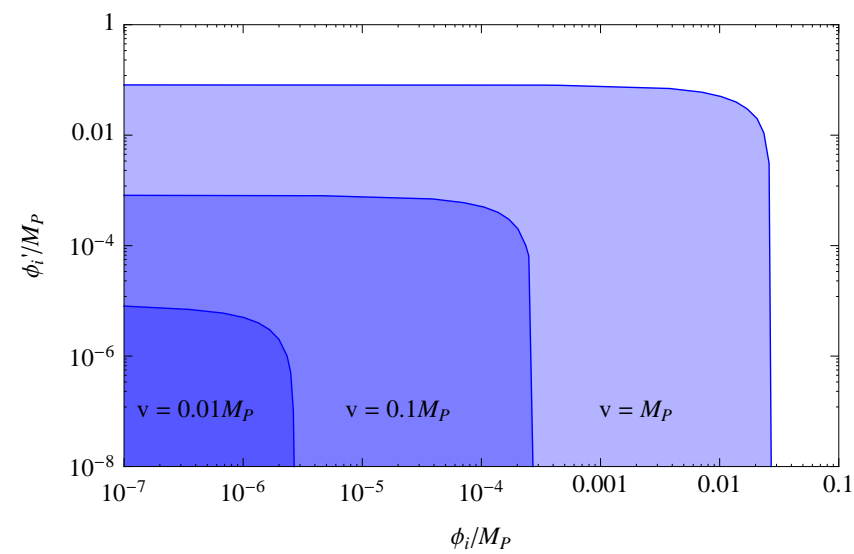

Figure 8. Regions in initial phase space $\left(\phi_{i}, \phi_{i}^{\prime}\right)$ that lead to over 60 e-folds of inflation for different values of $v \leq M_{P}$.

We show in Fig. 9 examples of phase space trajectories for the non-renormalizable potential with two different values of $v$. We use a re-scaled cosmic time $\tilde{t}=\sqrt{\lambda} t$, where $\lambda=V_{0} / v^{4}$.

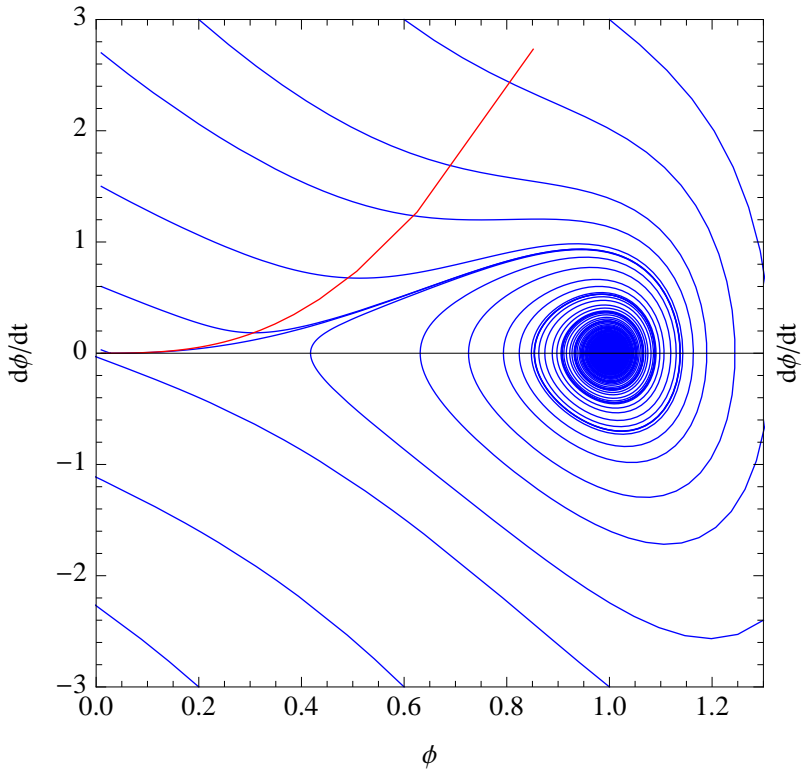

(a)

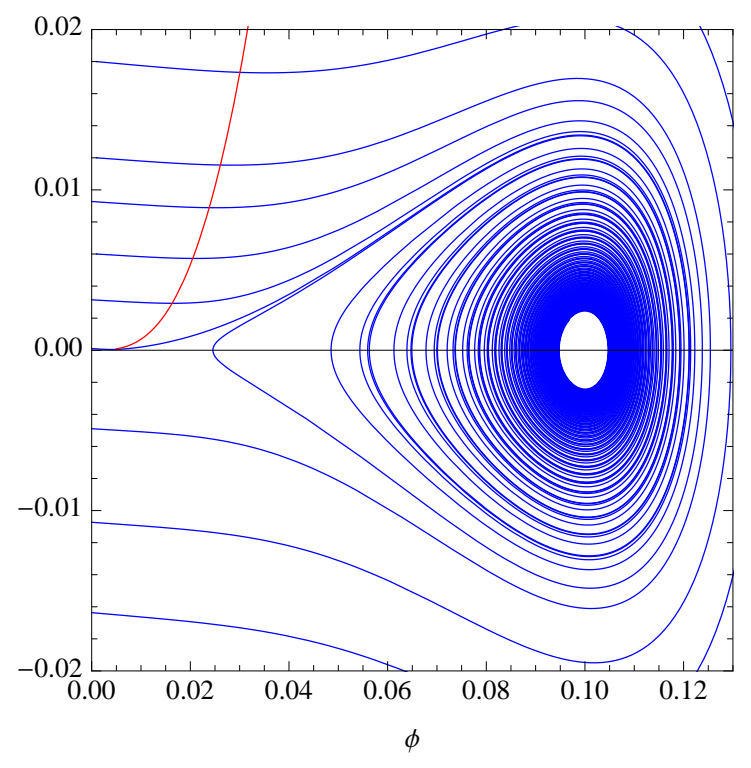

(b)

Figure 9. Phase space trajectories (shown in $M_{P}$ units) for the non-renormalizable plateau-like potential with $v=M_{P}$, panel (a) and for $v=0.1 M_{P}$, panel (b). Time is rescaled by a factor $\sqrt{\lambda}$ with respect to cosmic time. The red line gives, in each case, the slow-roll trajectory. 
Figure 9 clearly shows that the slow-roll solution is not a (global) attractor for $v<M_{P}$, but that it is nevertheless followed for sufficiently small values of the field and velocity as obtained above. Note that for the example with $v=0.1 M_{P}$, panel (b) in Fig. 9, there are trajectories that oscillate between the two minima before settling into one of them. This, of course, also occurs for any $v=M_{P}$ (and other values) but only for larger values of the velocity than shown in this example.

\section{B.2 General non-renormalizable plateau-like potentials}

Complementing the result of the previous subsection, we may consider a generic class of non-renormalizable plateau-like potentials of the form of Eq. (B.1) with arbitrary (integer) value $m \geq 6$. As illustrated in Fig. 10, for the case $m=n+2$, these potentials yield wider plateaux the larger the power $n$. In particular, the plateau region extends all the way to the minima in the limit $n \rightarrow+\infty$.

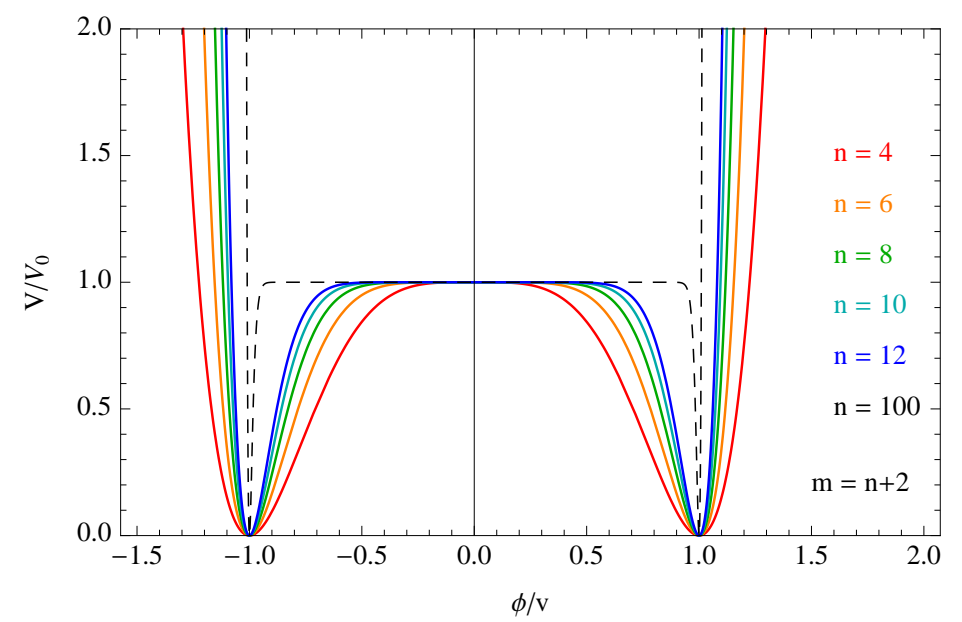

Figure 10. Non-renormalizable plateau-like potentials with different leading powers $n$ for $m=n+2$.

For such potentials, we have the slow-roll parameters

$$
\begin{aligned}
\epsilon_{\phi} & =\frac{1}{2}\left(\frac{M_{P}}{v}\right)^{2}\left(\frac{m n}{m-n}\right)^{2} x^{2 n-2}\left(\frac{x^{m-n}-1}{1-\frac{m}{m-n} x^{n}+\frac{n}{m-n} x^{m}}\right)^{2}, \\
\eta_{\phi} & =\left(\frac{M_{P}}{v}\right)^{2}\left(\frac{m n}{m-n}\right) x^{n-2}\left[\frac{1-n+(m-1) x^{m-n}-1}{1-\frac{m}{m-n} x^{n}+\frac{n}{m-n} x^{m}}\right] .
\end{aligned}
$$

The number of e-folds of inflation can be written in closed form as

$$
\begin{aligned}
N_{e} & =-\frac{1}{M_{P}^{2}} \int_{\phi_{*}}^{\phi_{e}} d \phi \frac{V(\phi)}{V^{\prime}(\phi)} \\
& =-\left.\frac{v^{2}}{M_{P}^{2}} \frac{1}{m n}\left\{\frac{m}{2} x^{2}-\mathrm{B}\left[x^{n-m}, \frac{(m-2)}{(m-n)}, 0\right]+\mathrm{B}\left[x^{n-m},-\frac{2}{(m-n)}, 0\right]\right\}\right|_{x_{*}} ^{x_{e}},
\end{aligned}
$$

where $\mathrm{B}(z, a, b)=\int_{0}^{z} t^{a-1}(1-t)^{b-1} d t$ is the incomplete beta-function [67] and $x_{e} \equiv \phi_{e} / v<1$ yields $\epsilon_{\phi}=1$. 
For the case $v \lesssim M_{P}$, we have $x_{*} \ll x_{e}<1$, and we may write the relevant observables and number of e-folds after horizon-crossing approximately as

$$
\begin{aligned}
n_{s} & =1-6 \epsilon_{\phi_{*}}+2 \eta_{\phi_{*}} \simeq 1-2(n-1)\left(\frac{m n}{m-n}\right)\left(\frac{M_{P}}{v}\right)^{2} x_{*}^{n-2}, \\
r & =16 \epsilon_{\phi_{*}} \simeq 8\left(\frac{m n}{m-n}\right)^{2}\left(\frac{M_{P}}{v}\right)^{2} x_{*}^{2 n-2}, \\
N_{e} & \simeq\left(\frac{m-n}{m n}\right)\left(\frac{v}{M_{P}}\right)^{2} \frac{1}{n-2} x_{*}^{2-n},
\end{aligned}
$$

such that we may write

$$
\begin{aligned}
n_{s} & \simeq 1-\frac{2}{N_{e}}\left(\frac{n-1}{n-2}\right) \\
r & \simeq 8\left(\frac{m n}{m-n}\right)^{-\frac{2}{n-2}}\left(\frac{v}{M_{P}}\right)^{\frac{2 n}{n-2}} \frac{1}{\left[(n-2) N_{e}\right]^{\frac{2 n-2}{n-2}}} .
\end{aligned}
$$

We see that the scalar spectral index is independent of the power $m$, while the tensor-to-scalar ratio exhibits a mild dependence on this exponent. For $n \gg 1$, we have

$$
n_{s} \simeq 1-\frac{2}{N_{e}}, \quad r \simeq \frac{8}{n^{2}}\left(\frac{v}{M_{P}}\right)^{2} \frac{1}{N_{e}^{2}} .
$$

We thus see that the large- $n$ prediction of this general class of potentials for the scalar spectral index coincides with that of the Starobinsky model [1], with $n_{s}=0.96-0.967$ for $50-60$ e-folds of inflation, which is in very good agreement with the Planck results. The tensor-to-scalar ratio is, in general, very suppressed for these potentials.

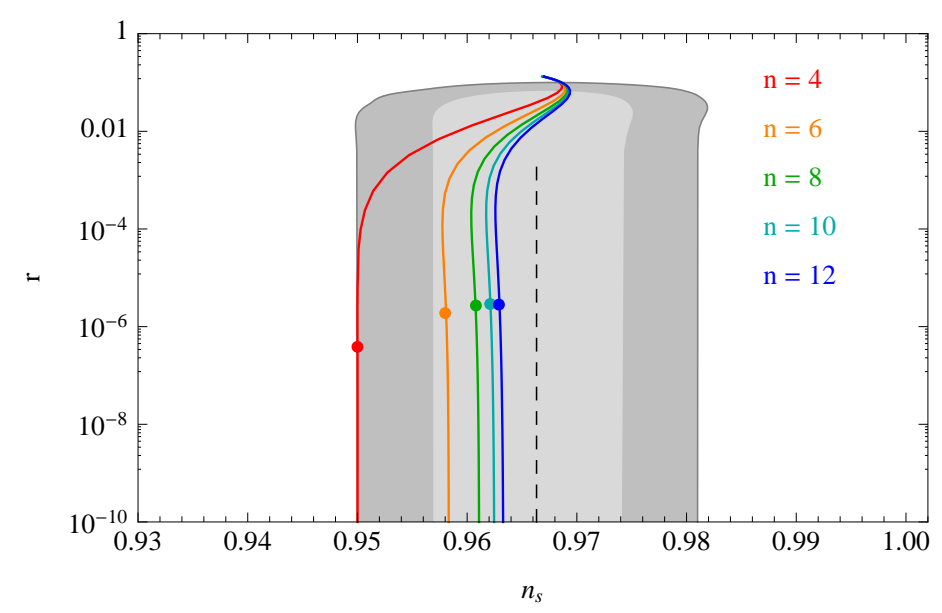

Figure 11. Observational predictions for non-renormalizable plateau-like potentials with different leading powers $n$ for $m=n+2$ and $N_{e}=60$. The ratio $v / M_{P}$ decreases from top to bottom in each curve. The circles give, in each case, the prediction for $v=M_{P}$ as a reference. The dashed black line gives the limiting prediction for $n_{s}$ with $v \lesssim M_{P}$.

In Fig. 11, we illustrate the predictions obtained for general values of $v / M_{P}$ with different powers $n$ for $m=n+2$ and $N_{e}=60$, where the approach to the Starobinsky value of $n_{s}$ is clear 
for $v \ll M_{P}$. We also see that the predictions for $v \gg M_{P}$ converge for the different values of $n$, and although the limiting value of $n_{s}$ is observationally consistent, the corresponding tensor-to-scalar ratio $r \simeq 0.13$ is already excluded.

This shows that, even though the quartic plateau potential is only marginally consistent with observational data, there exist similar potentials that are in perfect agreement with Planck in a sub-planckian regime. These potentials exhibit, moreover, a wider plateau and hence are less constraining in terms of initial conditions that can lead to a sufficiently long inflationary period. Our earlier analysis of initial conditions for the quartic plateau provides, thus, a worst-case scenario. Such non-renormalizable potentials should be, for example, ubiquitous in supersymmetric theories, where are there are several flat directions in scalar field space at the renormalizable level and which are only lifted by non-renormalizable terms.

\section{B.3 Non-renormalizable plateau model in warm inflation}

For completeness, we analyze the inflationary dynamics with the non-renormalizable plateau potential (B.2) in the case where dissipative effects cannot be neglected during the slow-roll phase. For a slowly rolling field, the main dissipative effects do not correspond to the field decay width as we have used for the oscillating field modes in the pre-inflationary radiation era, but arise from adiabatic dissipation that is non-vanishing at finite temperature [47]. In fact, such dissipative effects transfer a part of the inflaton's (kinetic) energy into the radiation bath, preventing it from being diluted. Hence, if there is sufficient dissipation in this adiabatic regime, a thermal bath can be sustained during inflation, leading to what is known as a warm rather than cold inflationary scenario.

The presence of the thermal bath must not, however, significantly alter the form of the inflaton potential during the slow-roll phase, and in particular the thermal mass that drove the evolution of the inflaton modes before the onset of inflation must become suppressed in the slow-roll regime. This may be possible if the fields coupled to the inflaton become nonrelativistic in the slow-roll phase, with $T \lesssim g \phi$ neglecting bare masses. Successful models of warm inflation in this low-temperature regime have been constructed in the context of supersymmetric models, leading to Yukawa interactions of the form we have considered in the pre-inflationary era and their scalar counter-parts. We have seen that supersymmetry may be indeed a way of controlling the flatness of the inflaton potential at zero-temperature and of keeping the inflaton self-coupling sufficiently small while allowing for significant interactions with other fields. The same is true at finite temperature in the low-temperature regime.

The superpotential yielding the relevant interactions is of the form

$$
W=\sum_{i, j}\left[g \Phi X_{i}^{2}+h X_{i} Y_{j}^{2}\right]
$$

where the inflaton is the scalar component of the $\Phi$ chiral superfield and the $N_{X}$ fermions and their scalar superpartners form the $X_{i}$ chiral superfields. The second term in the superpotential containing the $Y_{j}$ chiral fields allows for the decay of the $X_{i}$ fields. The mass of the $Y_{j}$ fields in the thermal bath does not depend on the inflaton field value and they can, thus, be kept light, while the $X_{i}$ fields become heavy in the slow-roll phase. Note that in the pre-inflationary era the inflaton is close to the origin and the $X_{i}$ fields are light, as assumed in our earlier analysis.

It has been shown that in the low-temperature regime the scalar components give the largest contribution to the adiabatic dissipation coefficient (namely from virtual modes), 
yielding a dissipation coefficient of the form $[47,52]$

$$
\Upsilon=C_{\phi} \frac{T^{3}}{\phi^{2}}, \quad C_{\phi}=0.04 h^{2} N_{Y} N_{X}
$$

We note that the corrections to the inflaton potential are determined by the t'Hooft coupling $g^{2} N_{X} \lesssim 1$ and that one also requires $h^{2} N_{Y} \lesssim 1$ for consistency of the perturbative calculation of the dissipation coefficient. Nevertheless, the constant $C_{\phi}$ can be made arbitrarily large by taking a large number of $X$ fields weakly coupled to the inflaton, i.e. $N_{X} \gg 1, g \ll 1$ while keeping $\alpha^{2} \sim g^{2} N_{X}$ with not too suppressed values as we have obtained earlier for thermalization of the inflaton field modes prior to inflation.

The dynamics of warm inflation with this dissipation coefficient is determined by the coupled system of inflaton and radiation equations along with the Friedmann equation, which in the slow-roll regime can be written as

$$
3 H(1+Q) \dot{\phi} \simeq-V^{\prime}(\phi), \quad \rho_{R} \simeq \frac{3}{4} Q \dot{\phi}^{2}, \quad H^{2} \simeq \frac{V(\phi)}{3 M_{P}^{2}},
$$

where $Q=\Upsilon / 3 H$. Consistency of the approximation requires in this case the slow-roll conditions $\epsilon_{\phi},\left|\eta_{\phi}\right|<1+Q$ to be satisfied. One can derive from these equations the evolution equation for the dissipative ratio $Q$ :

$$
\frac{Q^{\prime}}{Q}=\frac{1}{1+7 Q}\left(10 \epsilon_{\phi}-6 \eta_{\phi}+8 \sigma_{\phi}\right)
$$

where prime denote e-fold derivative and we have defined the additional slow-roll parameter $\sigma_{\phi}=M_{P}^{2}\left(V^{\prime} / V \phi\right)<1+Q$.

For the non-renormalizable plateau potential with sextic power, the slow-roll parameters are given in Eq. (B.3), which together with

$$
\sigma_{\phi} \simeq-12\left(\frac{M_{P}}{v}\right)^{2} \frac{x^{2}\left(1-x^{2}\right)}{1-3 x^{4}+2 x^{6}}
$$

allows us to write the slow-roll equation for $Q$ as

$$
\frac{Q^{\prime}}{Q} \simeq \frac{120}{1+7 Q}\left(\frac{M_{P}}{v}\right)^{2} x^{2}
$$

for $x=\phi / v \ll 1$. Therefore $Q^{\prime}>0$ and the dissipative ratio grows during inflation. Similarly, using the form of the dissipation coefficient and the slow-roll equation for $Q$, one obtains for the ratio $T / H$ :

$$
\frac{d \ln (T / H)}{d N_{e}}=\frac{2}{1+7 Q}\left[\frac{2+4 Q}{1+Q} \epsilon_{\phi}-\eta_{\phi}+\frac{1-Q}{1+Q} \sigma_{\phi}\right] \simeq \frac{48}{1+7 Q}\left(\frac{M_{P}}{v}\right)^{2} x^{2}\left(\frac{1+2 Q}{1+Q}\right)
$$

such that also the ratio $T / H$ grows during warm inflation for the non-renormalizable plateau potential, implying that inflation will occur in the warm regime if $T_{*} \gtrsim H_{*}$.

From the slow-roll Eqs. (B.13) one can derive a relation between the inflation field and $Q$ [57], and with the help of that to integrate Eq. (B.16) to obtain the number of e-folds. 
Since $Q^{\prime}>0$, we may consider the case where $Q_{*} \ll 1$ and $Q_{e} \gg 1$, which gives to leading order

$$
N_{e} \simeq \frac{1}{24}\left(\frac{v}{M_{P}}\right)^{2} x_{*}^{-2}\left(1+2.8 Q_{*}^{1 / 5}\right) .
$$

The case $Q_{*} \ll 1$ is particularly relevant for computing the spectrum of curvature perturbations generated during inflation, since in this regime one may neglect the coupling between inflaton and radiation fluctuations. In the warm regime $T_{*} \gtrsim H_{*}$, for thermal inflaton fluctuations the dimensionless power spectrum has been shown to take the form [33, 34, 46, 68, 69]

$$
\Delta_{\mathcal{R}}^{2}=\frac{1}{12 \pi^{2}} \epsilon_{\phi_{*}}^{-1} \frac{V_{*}}{M_{P}^{4}}\left(\frac{T_{*}}{H_{*}}\right),
$$

which differs from the standard cold scenario by a factor $2 T_{*} / H_{*}$, which therefore enhances the curvature power spectrum. To obtain the scalar spectral index one must differentiate as usual the above expression with respect to the number of e-folds of inflation (equivalent to the logarithmic differentiation with respect to the comoving wave number $k$ ). Using Eqs. (B.16) and (B.17), we have for the scalar spectral index

$$
n_{s}-1=2 \sigma_{\phi_{*}}-2 \epsilon_{\phi_{*}} \simeq-24\left(\frac{M_{P}}{v}\right)^{2} x_{*}^{2} \simeq-\frac{1}{N_{e}}\left(1+2.8 Q_{*}^{1 / 5}\right),
$$

which is red-tilted. In fact, for 50-60 e-folds of inflation one obtains $n_{s} \simeq 0.96-0.97$ for $Q_{*} \simeq 10^{-4}-10^{-2} \ll 1$ as assumed, showing that dissipative effects during inflation may bring this model into agreement with the Planck data even if they are still small at the time when the relevant CMB scales cross the horizon.

Since tensor modes are unaffected by the thermal bath during inflation, we have for the tensor-to-scalar ratio

$$
r=\frac{8 \epsilon_{\phi_{*}}}{T_{*} / H_{*}} \simeq 3 \times 10^{-6}\left(\frac{1-n_{s}}{0.04}\right)^{3}\left(\frac{v}{M_{P}}\right)^{4}\left(\frac{T_{*}}{H_{*}}\right)^{-1},
$$

which is extremely small, even smaller than in the corresponding supercooled inflationary scenario. Notice that this yields a modified consistency relation for warm inflation $r=$ $4\left|n_{t}\right| /\left(T_{*} / H_{*}\right)$ [26], but the smallness of the tensor-to-scalar ratio in this case implies that this may be very difficult to measure in the near future.

Let us check the consistency of the approximations made in the above calculations. First, we assumed that $Q_{e} \gg 1$ at the end of inflation. From $\left|\eta_{\phi}\right|=1+Q_{e} \simeq Q_{e}$ and the relation between $\phi$ and $Q$ we obtain that

$$
Q_{e} \simeq 36\left(\frac{0.04}{1-n_{s}}\right)^{5 / 2}\left(\frac{Q_{*}}{10^{-3}}\right)^{1 / 2},
$$

such that as assumed inflation ends in the strong dissipation regime. Secondly, we have assumed $x \equiv \phi / v \ll 1$ for the entire duration of inflation. From Eq. (B.20) and $\left|\eta_{\phi}\right| \simeq Q_{e}$ we have, respectively,

$$
x_{*} \simeq\left(\frac{1-n_{s}}{24}\right)^{1 / 2}\left(\frac{v}{M_{P}}\right) \simeq 0.04\left(\frac{v}{M_{P}}\right), \quad x_{e} \simeq\left(\frac{0.04}{1-n_{s}}\right)^{5 / 4}\left(\frac{Q_{*}}{10^{-3}}\right)^{1 / 4}\left(\frac{v}{M_{P}}\right)_{\mathrm{B},}
$$


such that this approximation is valid for $v<M_{P}$.

Finally, we assumed that the fields coupled to the inflaton are non-relativistic, $g \phi<T$, so as to suppress thermal corrections to the inflaton mass in the slow-roll regime. Using the slow-roll equations, one can show that

$$
\frac{d \log (\phi / T)}{d N_{e}} \simeq-\frac{12}{1+7 Q}\left(\frac{M_{P}}{v}\right)^{2} x^{2}\left(\frac{3+Q}{1+Q}\right)<0,
$$

such that $g \phi / T$ decreases during inflation. At the end of the slow-roll regime, we find:

$$
\frac{\phi_{e}}{T_{e}} \simeq 7435\left(\frac{g_{*}}{100}\right)^{1 / 6}\left(\frac{0.04}{1-n_{s}}\right)^{3 / 4}\left(\frac{Q_{*}}{10^{-3}}\right)^{1 / 12},
$$

such that we require couplings $g \gtrsim 10^{-4}$ to keep $g \phi \gtrsim T$ throughout inflation. This is compatible with a number of species $N_{X} \sim 10^{6}-10^{7}$ while keeping $g^{2} N_{X} \lesssim 1$ to avoid large quantum corrections to the inflaton potential.

\section{References}

[1] R. Brout, F. Englert and E. Gunzig, The Causal Universe, Gen. Rel. Grav. 10, 1 (1979);

R. Brout, F. Englert and E. Gunzig, The Creation Of The Universe As A Quantum

Phenomenon, Annals Phys. 115, 78 (1978);

A. A. Starobinsky, A New Type Of Isotropic Cosmological Models Without Singularity, Phys.

Lett. B 91, 99 (1980);

D. Kazanas, Dynamics of the Universe and Spontaneous Symmetry Breaking, Astrophys. J.

241, L59 (1980);

A. H. Guth, The Inflationary Universe: A Possible Solution To The Horizon And Flatness

Problems, Phys. Rev. D 23, 347 (1981);

K. Sato, First Order Phase Transition Of A Vacuum And Expansion Of The Universe, Mon.

Not. Roy. Astron. Soc. 195, 467 (1981);

L. Z. Fang, Entropy Generation in the Early Universe by Dissipative Processes Near the Higgs' Phase Transitions, Phys. Lett. B 95, 154 (1980).

[2] V. Mukhanov and G. Chibisov, Quantum Fluctuation And Nonsingular Universe. (In Russian), JETP Lett. 33, 532 (1981) [Pisma Zh. Eksp. Teor. Fiz. 33, 549 (1981)].

[3] G. Hinshaw et al. [WMAP Collaboration], Nine-Year Wilkinson Microwave Anisotropy Probe (WMAP) Observations: Cosmological Parameter Results, Astrophys. J. Suppl. 208, 19 (2013) doi:10.1088/0067-0049/208/2/19 [arXiv:1212.5226 [astro-ph.CO]].

[4] P. A. R. Ade et al. [Planck Collaboration], Planck 2015 results. XX. Constraints on inflation, Astron. Astrophys. 594, A20 (2016) doi:10.1051/0004-6361/201525898 [arXiv:1502.02114 [astro-ph.CO]].

[5] J. Khoury, B. A. Ovrut, P. J. Steinhardt and N. Turok, The Ekpyrotic universe: Colliding branes and the origin of the hot big bang, Phys. Rev. D 64, 123522 (2001) [hep-th/0103239].

[6] R. H. Brandenberger and C. Vafa, Superstrings In The Early Universe, Nucl. Phys. B 316, 391 (1989);

A. Nayeri, R. H. Brandenberger and C. Vafa, Producing a scale-invariant spectrum of perturbations in a Hagedorn phase of string cosmology, Phys. Rev. Lett. 97, 021302 (2006) [arXiv:hep-th/0511140];

R. H. Brandenberger, A. Nayeri, S. P. Patil and C. Vafa, String gas cosmology and structure formation, Int. J. Mod. Phys. A 22, 3621 (2007) [hep-th/0608121]. 
[7] F. Finelli and R. Brandenberger, On the generation of a scale-invariant spectrum of adiabatic fluctuations in cosmological models with a contracting phase, Phys. Rev. D 65, 103522 (2002) [arXiv:hep-th/0112249].

[8] D. Wands, Duality invariance of cosmological perturbation spectra, Phys. Rev. D 60, 023507 (1999) [gr-qc/9809062].

[9] R. Penrose, Difficulties with inflationary cosmology, Annals N. Y. Acad. Sci. 571, 249 (1989).

[10] G. W. Gibbons and N. Turok, The Measure Problem in Cosmology, Phys. Rev. D 77, 063516 (2008) [hep-th/0609095];

S. M. Carroll and H. Tam, Unitary Evolution and Cosmological Fine-Tuning, arXiv:1007.1417 [hep-th].

[11] T. Vachaspati and M. Trodden, Causality and cosmic inflation, Phys. Rev. D 61, 023502 (1999) [gr-qc/9811037];

L. Berezhiani and M. Trodden, How Likely are Constituent Quanta to Initiate Inflation?, Phys. Lett. B 749, 425 (2015) [arXiv:1504.01730 [hep-th]].

[12] A. Ijjas, P. J. Steinhardt and A. Loeb, Inflationary paradigm in trouble after Planck2013, Phys. Lett. B 723, 261 (2013) [arXiv:1304.2785 [astro-ph.CO]];

A. Ijjas, P. J. Steinhardt and A. Loeb, Inflationary schism after Planck2013, Phys. Lett. B 736, 142 (2014) [arXiv:1402.6980 [astro-ph.CO]].

[13] D. S. Goldwirth and T. Piran, Inhomogeneity and the Onset of Inflation, Phys. Rev. Lett. 64, 2852 (1990); D. S. Goldwirth and T. Piran, Initial conditions for inflation, Phys. Rept. 214, 223 (1992).

[14] A. Albrecht and R. H. Brandenberger, On the Realization of New Inflation, Phys. Rev. D 31, 1225 (1985); A. Albrecht, R. H. Brandenberger and R. Matzner, Numerical Analysis of Inflation, Phys. Rev. D 32, 1280 (1985); A. Albrecht, R. H. Brandenberger and R. Matzner, Inflation With Generalized Initial Conditions, Phys. Rev. D 35, 429 (1987).

[15] R. H. Brandenberger and J. H. Kung, Chaotic Inflation as an Attractor in Initial Condition Space, Phys. Rev. D 42, 1008 (1990).

[16] H. A. Feldman and R. H. Brandenberger, Chaotic Inflation With Metric and Matter Perturbations, Phys. Lett. B 227, 359 (1989). R. H. Brandenberger and H. A. Feldman, Effects of Gravitational Perturbations on the Evolution of Scalar Fields in the Early Universe, Phys. Lett. B 220, 361 (1989).

[17] R. Brandenberger, Initial Conditions for Inflation - A Short Review, arXiv:1601.01918 [hep-th].

[18] H. Kurki-Suonio, R. A. Matzner, J. Centrella and J. R. Wilson, Inflation From Inhomogeneous Initial Data in a One-dimensional Back Reacting Cosmology, Phys. Rev. D 35, 435 (1987) P. Laguna, H. Kurki- Suonio and R. A. Matzner, Inhomogeneous inflation: The Initial value problem, Phys. Rev. D 44, 3077 (1991). H. Kurki-Suonio, P. Laguna and R. A. Matzner, Inhomogeneous inflation: Numerical evolution, Phys. Rev. D 48, 3611 (1993) [astro-ph/9306009].

[19] W. E. East, M. Kleban, A. Linde and L. Senatore, Beginning inflation in an inhomogeneous universe, JCAP 1609, no. 09, 010 (2016) [arXiv:1511.05143 [hep-th]].

[20] K. Clough, P. Figueras, H. Finkel, M. Kunesch, E. A. Lim and S. Tunyasuvunakool, GRChombo : Numerical Relativity with Adaptive Mesh Refinement, Class. Quant. Grav. 32, no. 24, 245011 (2015) [arXiv:1503.03436 [gr-qc]];

K. Clough, E. A. Lim, B. S. DiNunno, W. Fischler, R. Flauger and S. Paban, Robustness of Inflation to Inhomogeneous Initial Conditions, arXiv:1608.04408 [hep-th].

[21] A. H. Guth, D. I. Kaiser and Y. Nomura, Inflationary paradigm after Planck 2013, Phys. Lett. B 733, 112 (2014) [arXiv:1312.7619 [astro-ph.CO]]. 
[22] A. Linde, Inflationary Cosmology after Planck 2013, arXiv:1402.0526 [hep-th].

[23] A. Berera and L. Z. Fang, Thermally induced density perturbations in the inflation era, Phys. Rev. Lett. 74, 1912 (1995) [astro-ph/9501024].

[24] A. Berera, Warm inflation, Phys. Rev. Lett. 75, 3218 (1995) [astro-ph/9509049].

[25] A. Berera, Thermal properties of an inflationary universe, Phys. Rev. D 54, 2519 (1996) [hep-th/9601134].

[26] S. Bartrum, M. Bastero-Gil, A. Berera, R. Cerezo, R. O. Ramos and J. G. Rosa, The importance of being warm (during inflation), Phys. Lett. B 732, 116 (2014) [arXiv:1307.5868 [hep-ph]].

[27] M. Bastero-Gil, A. Berera, R. O. Ramos and J. G. Rosa, Warm Little Inflaton, Phys. Rev. Lett. 117, no. 15, 151301 (2016) [arXiv:1604.08838 [hep-ph]].

[28] S. Bartrum, A. Berera and J. G. Rosa, Fluctuation-dissipation dynamics of cosmological scalar fields, Phys. Rev. D 91, no. 8, 083540 (2015) [arXiv:1412.5489 [hep-ph]].

[29] M. Bastero-Gil, A. Berera, R. O. Ramos and J. G. Rosa, Warm baryogenesis, Phys. Lett. B 712, 425 (2012) [arXiv:1110.3971 [hep-ph]].

[30] C. -H. Wu, K. -W. Ng, W. Lee, D. -S. Lee and Y. -Y. Charng, Quantum noise and a low cosmic microwave background quadrupole, J. Cosmol. Astropart. Phys. 02, 006 (2007). [astro-ph/0604292].

[31] W. Lee, K. -W. Ng, I-C. Wang and C. -H. Wu, Trapping effects on inflation, Phys. Rev. D 84, 063527 (2011). [arXiv:1101.4493 [hep-th]].

[32] A. Berera and R. Rangarajan, Quantum phase of inflation, Phys. Rev. D 87, 043514 (2013) Erratum: [Phys. Rev. D 87, 049901 (2013)] [arXiv:0912.5148 [astro-ph.CO]].

[33] R. O. Ramos and L. A. da Silva, Power spectrum for inflation models with quantum and thermal noises, JCAP 03 (2013) 032.

[34] M. Bastero-Gil, A. Berera, I. G. Moss and R. O. Ramos, Cosmological fluctuations of a random field and radiation fluid, JCAP 05 (2014) 004.

[35] M. Bastero-Gil, A. Berera, I. G. Moss and R. O. Ramos, Theory of non-Gaussianity in warm inflation, JCAP 12 (2014) 008.

[36] G. S. Vicente, L. A. da Silva and R. O. Ramos, Eternal inflation in a dissipative and radiation environment: Heated demise of eternity, Phys. Rev. D 93, no. 6, 063509 (2016).

[37] A. Berera and C. Gordon, Inflationary initial conditions consistent with causality, Phys. Rev. D 63, 063505 (2001) [hep-ph/0010280].

[38] E. Calzetta and M. Sakellariadou, Semiclassical effects and the onset of inflation, Phys. Rev. D 47, 3184 (1993) [arXiv:gr-qc/9209007]; C. Appignani and R. Casadio, A Radiation-like era before inflation, JCAP 0810, 027 (2008) [arXiv:0808.0092 [gr-qc]]; R. Dong, W. H. Kinney and D. Stojkovic, Symmetron Inflation, JCAP 1401, 021 (2014) [arXiv:1307.4451 [astro-ph.CO]].

[39] P. D. B. Collins and R. F. Langbein, On the thermodynamics of inflation, Phys. Rev. D 45, 3429 (1992).

[40] J. J. M. Carrasco, R. Kallosh and A. Linde, Cosmological Attractors and Initial Conditions for Inflation, Phys. Rev. D 92, no. 6, 063519 (2015) [arXiv:1506.00936 [hep-th]].

[41] M. Artymowski, Z. Lalak and M. Lewicki, Multi-phase induced inflation in theories with non-minimal coupling to gravity, arXiv:1607.01803 [astro-ph.CO].

[42] K. Dimopoulos and M. Artymowski, Initial conditions for inflation, arXiv:1610.06192 [astro-ph.CO]. 
[43] M. Le Bellac, Thermal field theory, Cambridge Monographs on Mathematical Physics (Cambridge University Press, Cambridge, UK, 1996).

[44] J. Yokoyama, Fate of oscillating scalar fields in the thermal bath and their cosmological implications, Phys. Rev. D 70, 103511 (2004) [hep-ph/0406072].

[45] M. Bastero-Gil, A. Berera and R. O. Ramos, Dissipation coefficients from scalar and fermion quantum field interactions, JCAP 1109, 033 (2011) [arXiv:1008.1929 [hep-ph]].

[46] A. Berera, Warm inflation at arbitrary adiabaticity: A Model, an existence proof for inflationary dynamics in quantum field theory, Nucl. Phys. B 585, 666 (2000) [hep-ph/9904409].

[47] A. Berera, I. G. Moss and R. O. Ramos, Warm Inflation and its Microphysical Basis, Rept. Prog. Phys. 72, 026901 (2009) [arXiv:0808.1855 [hep-ph]].

[48] N. G. van Kampen, Stochastic Processes in Physics and Chemistry, 2nd ed. (North-Holland, Amsterdam, 1992).

[49] V. F. Mukhanov, H. A. Feldman and R. H. Brandenberger, Theory of cosmological perturbations. Part 1. Classical perturbations. Part 2. Quantum theory of perturbations. Part 3. Extensions, Phys. Rept. 215, 203 (1992).

[50] J. H. Kung and R. H. Brandenberger, The Initial Condition Dependence of Inflationary Universe Models, Phys. Rev. D 40, 2532 (1989).

[51] R. H. Brandenberger, H. Feldman and J. Kung, Initial conditions for chaotic inflation, Phys. Scripta T 36, 64 (1991).

[52] M. Bastero-Gil, A. Berera, R. O. Ramos and J. G. Rosa, General dissipation coefficient in low-temperature warm inflation, JCAP 1301, 016 (2013) [arXiv:1207.0445 [hep-ph]].

[53] T. Appelquist and J. Carazzone, Infrared Singularities and Massive Fields, Phys. Rev. D 11, 2856 (1975).

[54] M. Bastero-Gil, A. Berera and B. M. Jackson, Power suppression from disparate mass scales in effective scalar field theories of inflation and quintessence, JCAP 1107, 010 (2011) [arXiv:1003.5636 [hep-ph]].

[55] M. Benetti and R. O. Ramos, Warm inflation dissipative effects: predictions and constraints from the Planck data, Phys. Rev. D 95, no. 2, 023517 (2017) [arXiv:1610.08758 [astro-ph.CO]].

[56] J. S. Borges and R. O. Ramos, Symmetry breaking patterns of the 3-3-1 model at finite temperature, Eur. Phys. J. C 76, no. 6, 344 (2016) [arXiv:1602.08165 [hep-ph]].

[57] M. Bastero-Gil and A. Berera, Warm inflation model building, Int. J. Mod. Phys. A 24, 2207 (2009) [arXiv:0902.0521 [hep-ph]].

[58] D. H. Lyth and E. D. Stewart, Cosmology with a TeV mass GUT Higgs, Phys. Rev. Lett. 75, 201 (1995) [hep-ph/9502417].

[59] D. H. Lyth and E. D. Stewart, Thermal inflation and the moduli problem, Phys. Rev. D 53, 1784 (1996) [hep-ph/9510204].

[60] J. I. Kapusta and C. Gale, Finite-Temperature Field Theory: Principles and Applications, (Cambridge University Press, Cambridge, England, 2006).

[61] J. H. Traschen and R. H. Brandenberger, Particle Production During Out-of-equilibrium Phase Transitions, Phys. Rev. D 42, 2491 (1990).

[62] R. Allahverdi, R. Brandenberger, F. Y. Cyr-Racine and A. Mazumdar, Reheating in Inflationary Cosmology: Theory and Applications, Ann. Rev. Nucl. Part. Sci. 60, 27 (2010) [arXiv:1001.2600 [hep-th]]. 
[63] M. A. Amin, M. P. Hertzberg, D. I. Kaiser and J. Karouby, Nonperturbative Dynamics Of Reheating After Inflation: A Review, Int. J. Mod. Phys. D 24, 1530003 (2014) [arXiv:1410.3808 [hep-ph]].

[64] A. Berera, L. Z. Fang and G. Hinshaw, An Attempt to determine the largest scale of primordial density perturbations in the universe, Phys. Rev. D 57, 2207 (1998) [astro-ph/9703020].

[65] K. Bhattacharya, S. Mohanty and R. Rangarajan, Temperature of the inflaton and duration of inflation from WMAP data, Phys. Rev. Lett. 96, 121302 (2006) [hep-ph/0508070].

[66] A. Kehagias and A. Riotto, Remarks about the Tensor Mode Detection by the BICEP2 Collaboration and the Super-Planckian Excursions of the Inflaton Field, Phys. Rev. D 89, no. 10, 101301 (2014) [arXiv:1403.4811 [astro-ph.CO]].

[67] Handbook of Mathematical Functions, edited by M. Abramowitz and I. A. Stegun (Dover, New York, 1972), 9th ed.

[68] L. M. H. Hall, I. G. Moss and A. Berera, Scalar perturbation spectra from warm inflation, Phys. Rev. D 69 (2004) 083525 [astro-ph/0305015].

[69] C. Graham and I. G. Moss, Density fluctuations from warm inflation, JCAP 0907 (2009) 013 [arXiv:0905.3500 [astro-ph.CO]]. 